Article

\title{
Clinical Characteristics and Disease Progression in Early-Stage COVID-19 Patients in South Korea
}

\author{
Min Hyuk Choi ${ }^{1,2,+} \oplus$, Hyunmin Ahn ${ }^{3,+}$, Han Seok Ryu ${ }^{4} \oplus$, Byung-Jun Kim ${ }^{4}$, Joonyong Jang ${ }^{4}$, \\ Moonki Jung ${ }^{4}$, Jinuoung Kim ${ }^{4}$ and Seok Hoon Jeong ${ }^{1, *(D)}$ \\ 1 Department of Laboratory Medicine and Research Institute of Bacterial Resistance, Yonsei University \\ College of Medicine, Seoul 06273, Korea; tcmhwd@yuhs.ac \\ 2 Department of Laboratory Medicine, Armed Forces Daegu Hospital, Daegu 712-906, Korea \\ 3 Department of Ophthalmology, Armed Forces Daegu Hospital, Daegu 712-906, Korea; overhyun@gmail.com \\ 4 Department of Internal Medicine, Armed Forces Daegu Hospital, Daegu 712-906, Korea; \\ cpcforever@nate.com (H.S.R.); rla20404@naver.com (B.-J.K.); zun54@naver.com (J.J.); \\ yessul88@naver.com (M.J.); jinote0504@gmail.com (J.K.) \\ * Correspondence: kscpjsh@yuhs.ac; Tel.: +82-2-2019-2776; Fax: +82-2-2057-8926 \\ + These authors contributed equally to this study.
}

Received: 3 June 2020; Accepted: 19 June 2020; Published: 23 June 2020

\begin{abstract}
A rapid increase in the number of patients with coronavirus disease 19 (COVID-19) may overwhelm the available medical resources. We aimed to evaluate risk factors for disease severity in the early stages of COVID-19. The cohort comprised 293 patients with COVID-19 from 5 March 2020, to 18 March 2020. The Korea Centers for Disease Control and Prevention (KCDC) classification system was used to triage patients. The clinical course was summarized, including the impact of drugs (angiotensin II receptor blockers [ARB], ibuprofen, and dipeptidyl peptidase-4 inhibitors [DPP4i]) and the therapeutic effect of lopinavir/ritonavir. After adjusting for confounding variables, prior history of drug use, including ARB, ibuprofen, and DPP4i was not a risk factor associated with disease progression. Patients treated with lopinavir/ritonavir had significantly shorter progression-free survival than those not receiving lopinavir/ritonavir. KCDC classification I clearly distinguished the improvement/stabilization group from the progression group of COVID-19 patients (AUC 0.817; 95\% CI, 0.740-0.895).
\end{abstract}

Keywords: coronavirus disease-19 (COVID-19); severe acute respiratory syndrome coronavirus 2 (SARS-CoV-2); angiotensin II receptor blockers (ARB); ibuprofen; Korea centers for disease control and prevention $(\mathrm{KCDC})$ classification

\section{Introduction}

Coronaviruses, enveloped viruses with a positive-sense single-stranded RNA genome, comprise the family Coronaviridae, order Nidovirales and are widely distributed in birds, humans, and other mammals [1]. The novel coronavirus disease (COVID-19) caused by severe acute respiratory syndrome coronavirus 2 (SARS-CoV-2) infection emerged in Wuhan, Hubei, China, on 8 December 2019 [2-4]. The outbreak has spread worldwide, and the number of confirmed cases is growing rapidly [5].

Most COVID-19 patients have mild symptoms, such as fever and cough [5], and have a favorable prognosis without specific treatment $[4,6,7]$. In severe cases, dyspnea and hypoxia may develop within one week after onset of the disease and may rapidly progress to acute respiratory distress syndrome (ARDS), acute respiratory failure, septic shock, metabolic acidosis, and coagulopathy [8].

The first case of COVID-19 in South Korea was a resident of Wuhan, China, who entered Incheon Airport on 19 January 2020 [9]. On 17 February, the 31st COVID-19 patient was confirmed to have 
participated in religious ceremonies in Daegu city. Subsequently, multiple COVID-19 outbreaks occurred in South Korea, including community-associated outbreaks in Daegu city and healthcare-associated outbreaks in Cheongdo, Gyeongsangbuk-do province [10].

A rapid increase in number of patients with COVID-19 can overwhelm the available medical resources, including intensive care units, negative pressure beds, and medical staff. Therefore, early assessment of risk factors for disease progression and patient prognosis is critical to ensure that patients whose disease is more likely to increase in severity can receive proper treatment in a timely manner. Thus, the Korea Centers for Disease Control and Prevention (KCDC) have established a system to triage patients in public health centers, whereby mild cases are transferred to living treatment centers, mild to moderate cases to dedicated cohort hospitals, and severe cases to tertiary university hospitals [11]. Large-scale diagnostic testing was performed to find hidden COVID-19 cases in sub-populations that had a history of contact with confirmed cases. Through these processes, South Korean patients with asymptomatic infection or early symptoms of disease were identified, and early monitoring and treatment of patients with COVID-19 were conducted.

Although many published studies have summarized the clinical features of COVID-19 patients [4,6,7,12-15], few have addressed the course of the disease in the early stages of symptom onset. Therefore, the clinical characteristics, imaging features, and treatment outcomes of COVID-19 patients before or immediately after onset of symptoms were investigated, with a particular focus on mild to moderate cases. We aimed to evaluate risk factors and KCDC classification models to predict disease progression in patients with early-stage COVID-19.

\section{Methods}

\subsection{Study Participants}

All consecutive patients with confirmed COVID-19 admitted to the Armed Forces Daegu Hospital, Daegu, South Korea, from 5 March 2020 to 18 March 2020, were enrolled in this study. According to the arrangement established by the government, the hospital was designated as a COVID-19-dedicated, 300-bed cohort hospital. The final follow-up date for this study was April 4, 2020. A total of 293 adult patients from Daegu city was admitted to the hospital during the study period. All cases were confirmed as COVID-19 using a real-time reverse transcription polymerase chain reaction (RT-PCR) (Seegene Inc., Seoul, South Korea, https://www.seegene.com) assay of nasal and oropharyngeal swabs [4]. This retrospective cohort study was approved by the Institutional Review Board of the Korean Military Medical Association (Seongnam-si, Gyeonggi-do, South Korea) (AFMC-20015-IRB-20-015).

The following clinical data were collected using electronic medical records: age at diagnosis, sex, signs and symptoms, date of symptom onset, date of hospital admission, date of discharge or transfer, Charlson comorbidity index [16], Eastern Cooperative Oncology Group (ECOG) performance status [17], Multilobular infiltration, hypo-Lymphocytosis, Bacterial coinfection, Smoking history, hyper-Tension and Age (MuLBSTA) score [18], pneumonia severity index [19], Confusion, Urea, Respiratory rate, Blood pressure plus age $\geq 65$ years (CURB-65) [20], respiratory support, and treatment agents administered before and during hospitalization. We also obtained radiologic findings. Baseline non-contrast-enhanced chest computed tomography (CT) was completed for all patients to assess disease severity. To ensure the safety of medical staff by minimizing contact with patients, routine laboratory tests were not conducted in all patients but only for patients requiring clinical decisions.

\subsection{Definitions}

The triage algorithm and classification criteria according to the COVID-19 response guidelines (version 7) developed by KCDC are presented in Figure 1. The criteria address the patient's mental state, age, history of underlying comorbidities, history of smoking, respiratory symptoms, and body temperature (BT). The "KCDC Classification I" was applied if the patient's blood pressure (BP), 
pulse rate (PR), and respiratory rate (RR) could not be measured, and "KCDC Classification II" was applied if these parameters could be measured; thereafter, patients with COVID-19 were classified into one of four groups (Class I to IV).
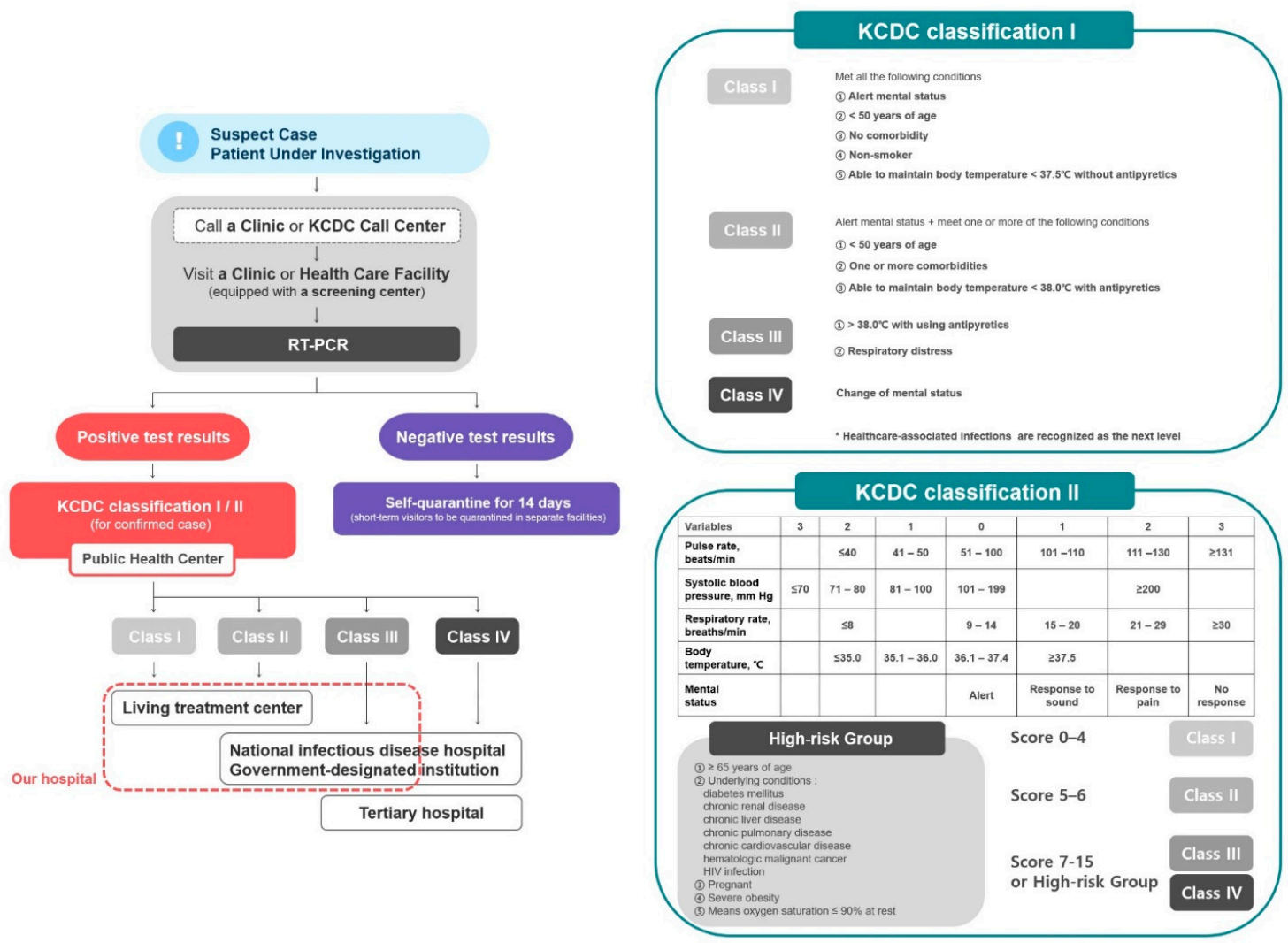

Figure 1. Triage algorithm and KCDC classification criteria for clinical severity of COVID-19 in South Korea. Abbreviations: KCDC, Korea Centers for Disease Control and Prevention; RT-PCR, real-time reverse transcription polymerase chain reaction Figure legend: The Living Treatment Center is a quarantine facility for mild or asymptomatic COVID-19 patients who are unable to self-isolate at home. The patients were checked for vital signs twice a day and immediately transferred to hospitals if their symptoms worsened. If their symptoms resolved, the patient was tested according to the standards for lifting the quarantine. Certain state-run facilities and accommodations are designated as Living Treatment Centers and are supplied with medical staff, medical equipment (pulse oximetry device, thermometer, blood pressure monitor, CPR kit, chest X-ray radiograph, etc.), individual relief kits (underwear, toiletries, face masks, etc.), and hygiene kits (thermometer and medical supplies).

In previous studies of COVID-19 patients [8,12], "mild cases" were defined as patients who experienced mild symptoms, with no manifestations of pneumonia on chest imaging. "Moderate cases" referred to patients with uncontrolled fever despite administration of antipyretics and/or respiratory symptoms. Severe cases of COVID-19 were defined as patients with any of the following: respiratory distress, $R R \geq 30$ breaths/min; mean oxygen saturation $\leq 93 \%$ at rest; arterial oxygen partial pressure/inspired oxygen fraction $\leq 300 \mathrm{~mm} \mathrm{Hg}$.

To quantify opacifications on pulmonary images, we applied the "CT score" as proposed in previous reports [21-23]. In brief, each pulmonary lobe was scored as 0 (none), 1 (diameter $<1 \mathrm{~cm}$ ), 2 (diameter 1 to $3 \mathrm{~cm}$ ), 3 (diameter $3 \mathrm{~cm}$ to $<50 \%$ of the lobe), or 4 ( $50 \%$ to $100 \%$ of the lobe) depending on lesion size and abnormal area. The overall score was calculated by summing all five lobar scores. 


\subsection{Outcomes}

Depending on the course of the disease during hospitalization, patients were classified into either the progression or improvement/stabilization group. The progression group comprised mild or moderate cases that progressed to moderate or severe cases, while the improvement/stabilization group comprised mild cases that did not progress further. Progression-free survival (PFS) was defined as the duration of time over which patients with COVID-19 remained stable during their hospitalization.

\subsection{Propensity Score (PS)-Matched Analyses}

To adjust the outcomes of patients with COVID-19 for potential confounding factors, we conducted a PS-matched case-control study. We selected 10 variables for adjustment using univariable analyses (Table A1): age, healthcare-associated infection, ECOG performance status, asymptomatic on initial evaluation, BT at hospital admission, diastolic BP at hospital admission, PR at hospital admission, $\mathrm{SpO}_{2}$ at hospital admission, hypertension, and diabetes mellitus [24,25]. We then performed PS-matched analyses by attempting to match cases and control patients (1:1 matching) using the nearest-neighbor-matching method. A match occurred when the difference in the logits of the PS was $<0.2$ times the standard deviation (SD) of the scores.

\subsection{Statistical Analysis}

We assessed all variables using the Shapiro-Wilk test to evaluate Gaussian distributions. Descriptive statistics are presented as median and interquartile range (IQR) for continuous and categorical variables. Comparisons between groups were analyzed using the Mann-Whitney $U$ test for continuous variables and Fisher's exact test for categorical variables. PFS was analyzed using the Kaplan-Meier method, and differences between groups were qualified by log-rank testing. To obtain ORs and hazard ratios (HRs), univariate regressions were performed using logistic and Cox regression, respectively. All reported $p$ values are two-tailed, and $p$ values $<0.05$ indicate statistical significance. We conducted statistical analyses using R statistical software (R Studio, Inc., https://www.r-project.org).

\section{Results}

\subsection{Characteristics of Patients}

\subsubsection{Before PS Matching}

The demographic and clinical characteristics of the progression and improvement/stabilization groups are summarized in Table 1. The median age of the 293 patients was 29 years (IQR, 24-47 years), and $214(73.0 \%)$ patients were male. Of the infections, $98.0 \%$ were community-associated cases. The most common symptoms at admission were productive cough (83 [28.3\%]), fever (75 [25.6\%]), and cough $(69[23.5 \%])$, but more patients were asymptomatic (97 [33.1\%]), and 279 patients were assigned ECOG performance status scores of zero (95.2\%).

The median days from the onset of symptoms to disease confirmation was 1 day (IQR, 0-6 days), and that from onset to hospital admission was 6 days (IQR, $0-12$ days). The median duration of hospitalization was 18 days (IQR, 15-20 days), and hospitalized patients had a median duration of symptoms of 7 days (IQR, 0-15 days). As of April 4, 2020, 207 (70.6\%) of 293 patients had been discharged, and $2(0.7 \%)$ patients had been transferred due to symptom aggravation. The patients' discharge assessments were based on abatement of all symptoms, with two consecutive negative RT-PCR tests for COVID-19.

According to baseline chest CT imaging, 64 (21.8\%) patients had findings consistent with bilateral pneumonia, and $56(19.1 \%)$ patients had unilateral pneumonia. Supplementary oxygen was required in 10 patients $(3.4 \%)$. One hundred patients $(34.1 \%)$ were administered antibiotics empirically: the treatment regimen was quinolone (84 patients [28.7\%]) or combination therapy with cefotaxime and doxycycline (14 patients [4.8\%]). In addition, 30 patients $(10.2 \%)$ received lopinavir/ritonavir 
antiviral therapy, although it was withdrawn in 21 of these 30 patients $(70.0 \%)$ due to side effects such as nausea and vomiting.

Thirty-six (12.3\%) cases were classified as the progression group, and the remaining 257 (87.7\%) cases were classified as the improvement/stabilization group. The progression group of COVID-19 patients was significantly older than the improvement/stabilization group (49.5 vs. 27.0 years of age; $p<0.001)$. There were no statistically significant differences between sex and times from symptom onset to confirmation/ admission. The progression group included a greater proportion of cases of healthcare-associated infection than the improvement/stabilization group $(p=0.003)$. A greater proportion of patients in the progression group presented initial symptoms of fever, chest pain, dyspnea, myalgia or fatigue, chills, and diarrhea compared with patients in the improvement/stabilization group, while a greater proportion of patients in the improvement/stabilization group were asymptomatic.

Compared with the improvement/stabilization group, the progression group was more likely to have comorbidities such as hypertension $(p=0.003)$ and diabetes mellitus $(p<0.001)$. Given the greater incidence of pre-existing conditions, a greater proportion of patients in the progression group had a history of drug use, including ibuprofen $(p=0.044)$, angiotensin II receptor blockers $(\mathrm{ARB} ; p=0.006)$, calcium channel blockers (CCB; $p=0.047)$, dipeptidyl peptidase-4 inhibitors (DPP4i; $p<0.001)$, metformin $(p<0.001)$, and/or statins $(p=0.006)$.

Table 1. Baseline characteristics of patients with COVID-19, before propensity-score matching *.

\begin{tabular}{|c|c|c|c|c|}
\hline & Total, No. (\%) & Progression, No. (\%) & $\begin{array}{c}\text { Improvement/ } \\
\text { Stabilization, No. }(\%)\end{array}$ & $p$ \\
\hline & $(n=293)$ & $(n=36)$ & $(n=257)$ & Value \\
\hline Age, median (IQR) & $29(24-47)$ & $49.5(34-57)$ & $27(23-46)$ & $<0.001$ \\
\hline Male sex & $214(73.0 \%)$ & $24(66.7 \%)$ & $190(73.9 \%)$ & 0.472 \\
\hline Healthcare-associated infection & $6(2.0 \%)$ & $3(8.3 \%)$ & $3(1.2 \%)$ & 0.027 \\
\hline ECOG performance status & & & & $<0.001$ \\
\hline 0 & $279(95.2 \%)$ & $28(77.8 \%)$ & $251(97.7 \%)$ & \\
\hline 1 & $12(4.1 \%)$ & $8(22.2 \%)$ & $4(1.6 \%)$ & \\
\hline 2 & $2(0.7 \%)$ & $0(0.0 \%)$ & $2(0.8 \%)$ & \\
\hline $\begin{array}{l}\text { Time from disease confirmation to admission, } \\
\text { median (IQR), days }\end{array}$ & $5.0(3.0-6.0)$ & $4.0(3.0-6.0)$ & $5.0(3.0-6.0)$ & 0.712 \\
\hline $\begin{array}{l}\text { Time from symptom onset to admission, } \\
\text { median (IQR), days }\end{array}$ & $6.0(0.0-12.0)$ & $8.0(3.0-10.5)$ & $5.0(0.0-12.0)$ & 0.156 \\
\hline $\begin{array}{l}\text { Time from symptom onset to confirmation, } \\
\text { median (IQR), days }\end{array}$ & $1.0(0.0-6.0)$ & $3.0(0.0-6.0)$ & $1.0(0.0-5.0)$ & 0.198 \\
\hline $\begin{array}{l}\text { Time from admission to discharge, median } \\
\text { (IQR), days }\end{array}$ & $18.0(15.0-20.0)$ & $18.0(14.0-20.0)$ & $18.0(15.5-21.0)$ & 0.568 \\
\hline Symptom duration, median (IQR), days & $7.0(0.0-15.0)$ & $12.0(10.0-20.0)$ & $6.0(0.0-15.0)$ & $<0.001$ \\
\hline \multicolumn{5}{|l|}{ Initial symptoms (may be multiple) } \\
\hline Asymptomatic & $97(33.1 \%)$ & $3(8.3 \%)$ & $94(36.6 \%)$ & 0.001 \\
\hline Productive cough & $83(28.3 \%)$ & $15(41.7 \%)$ & $68(26.5 \%)$ & 0.089 \\
\hline Fever & $75(25.6 \%)$ & $20(55.6 \%)$ & $55(21.4 \%)$ & $<0.001$ \\
\hline Cough & $69(23.5 \%)$ & $4(11.1 \%)$ & $65(25.3 \%)$ & 0.095 \\
\hline Headache & $61(20.8 \%)$ & $16(44.4 \%)$ & $45(17.5 \%)$ & $<0.001$ \\
\hline Myalgia or fatigue & $60(20.5 \%)$ & $14(38.9 \%)$ & $46(17.9 \%)$ & 0.007 \\
\hline Chills & $54(18.4 \%)$ & $19(52.8 \%)$ & $35(13.6 \%)$ & $<0.001$ \\
\hline Sore throat & $42(14.3 \%)$ & $7(19.4 \%)$ & $35(13.6 \%)$ & 0.496 \\
\hline Rhinorrhea & $36(12.3 \%)$ & $4(11.1 \%)$ & $32(12.5 \%)$ & $>0.999$ \\
\hline Dyspnea & $22(7.5 \%)$ & $10(27.8 \%)$ & $12(4.7 \%)$ & $<0.001$ \\
\hline Diarrhea & $19(6.5 \%)$ & $7(19.4 \%)$ & $12(4.7 \%)$ & 0.003 \\
\hline Nausea or vomiting & $9(3.1 \%)$ & $3(8.3 \%)$ & $6(2.3 \%)$ & 0.150 \\
\hline
\end{tabular}


Table 1. Cont.

\begin{tabular}{|c|c|c|c|c|}
\hline & Total, No. (\%) & Progression, No. $(\%)$ & $\begin{array}{c}\text { Improvement/ } \\
\text { Stabilization, No. (\%) }\end{array}$ & $p$ \\
\hline & $(n=293)$ & $(n=36)$ & $(n=257)$ & Value \\
\hline Chest pain & $9(3.1 \%)$ & $4(11.1 \%)$ & $5(1.9 \%)$ & 0.014 \\
\hline Other & $8(2.7 \%)$ & $1(2.8 \%)$ & $7(2.7 \%)$ & $>0.999$ \\
\hline \multicolumn{5}{|l|}{ Initial signs (may be multiple) } \\
\hline Body temperature, median (IQR), ${ }^{\circ} \mathrm{C}$ & $36.7(36.5-37.0)$ & $37.3(36.5-37.7)$ & $36.7(36.5-37.0)$ & $<0.001$ \\
\hline Systolic blood pressure, median (IQR), $\mathrm{mm} \mathrm{Hg}$ & $\begin{array}{c}133.0 \\
(121.0-141.0)\end{array}$ & $135.5(121.5-146.0)$ & $132.0(121.0-141.0)$ & 0.335 \\
\hline Diastolic blood pressure, median (IQR), $\mathrm{mm} \mathrm{Hg}$ & $79.0(72.0-85.0)$ & $82.0(75.5-90.5)$ & $78.0(72.0-84.0)$ & 0.015 \\
\hline Pulse rate, median (IQR), beats/min & $86.0(76.0-96.0)$ & $90.0(79.0-102.5)$ & $85.0(75.0-95.0)$ & 0.010 \\
\hline Respiratory rate, median (IQR), breaths/min & $16.0(16.0-18.0)$ & $16.0(16.0-18.0)$ & $16.0(16.0-18.0)$ & 0.105 \\
\hline SpO2, median (IQR), \% & $98.0(98.0-99.0)$ & $98.0(97.0-99.0)$ & $98.0(98.0-99.0)$ & 0.023 \\
\hline \multicolumn{5}{|l|}{ Comorbidities (may be multiple) } \\
\hline Hypertension & $29(9.9 \%)$ & $9(25.0 \%)$ & $20(7.8 \%)$ & 0.003 \\
\hline Diabetes mellitus & $21(7.2 \%)$ & $10(27.8 \%)$ & $11(4.3 \%)$ & $<0.001$ \\
\hline Allergic disease & $38(13.0 \%)$ & $1(2.8 \%)$ & $37(14.4 \%)$ & 0.093 \\
\hline Chronic lung disease & $17(5.8 \%)$ & $3(8.3 \%)$ & $14(5.4 \%)$ & 0.754 \\
\hline Peripheral vascular disease & $13(4.4 \%)$ & $1(2.8 \%)$ & $12(4.7 \%)$ & 0.933 \\
\hline Malignant tumor(s) & $7(2.4 \%)$ & $0(0.0 \%)$ & $7(2.7 \%)$ & 0.675 \\
\hline Liver disease & $5(1.7 \%)$ & $1(2.8 \%)$ & $4(1.6 \%)$ & $>0.999$ \\
\hline Congestive heart failure & $6(2.0 \%)$ & $2(5.6 \%)$ & $4(1.6 \%)$ & 0.338 \\
\hline Cerebrovascular disease & $5(1.7 \%)$ & $2(5.6 \%)$ & $3(1.2 \%)$ & 0.224 \\
\hline Rheumatic disease & $2(0.7 \%)$ & $1(2.8 \%)$ & $1(0.4 \%)$ & 0.583 \\
\hline Acute myocardial infarction & $1(0.3 \%)$ & $0(0.0 \%)$ & $1(0.4 \%)$ & $>0.999$ \\
\hline Kidney disease & $1(0.3 \%)$ & $0(0.0 \%)$ & $1(0.4 \%)$ & $>0.999$ \\
\hline \multicolumn{5}{|l|}{ Prior history of drug use } \\
\hline Ibuprofen & $21(7.2 \%)$ & $6(16.7 \%)$ & $15(5.8 \%)$ & 0.044 \\
\hline Angiotensin II receptor blockers & $16(5.5 \%)$ & $6(16.7 \%)$ & $10(3.9 \%)$ & 0.006 \\
\hline Calcium channel blocker & $16(5.5 \%)$ & $5(13.9 \%)$ & $11(4.3 \%)$ & 0.047 \\
\hline Beta blocker & $9(3.1 \%)$ & $2(5.6 \%)$ & $7(2.7 \%)$ & 0.684 \\
\hline Diuretics & $2(0.7 \%)$ & $0(0.0 \%)$ & $2(0.8 \%)$ & $>0.999$ \\
\hline Dipeptidyl peptidase- 4 inhibitor & $16(5.5 \%)$ & $8(22.2 \%)$ & $8(3.1 \%)$ & $<0.001$ \\
\hline Metformin & $12(4.1 \%)$ & $6(16.7 \%)$ & $6(2.3 \%)$ & $<0.001$ \\
\hline Sulfonylurea & $4(1.4 \%)$ & $2(5.6 \%)$ & $2(0.8 \%)$ & 0.122 \\
\hline Thiazolidinedione & $1(0.3 \%)$ & $1(2.8 \%)$ & $0(0.0 \%)$ & 0.250 \\
\hline Sodium-glucose cotransporter-2 inhibitor & $1(0.3 \%)$ & $0(0.0 \%)$ & $1(0.4 \%)$ & $>0.999$ \\
\hline Gabapentinoid & $1(0.3 \%)$ & $0(0.0 \%)$ & $1(0.4 \%)$ & $>0.999$ \\
\hline Isosorbide & $1(0.3 \%)$ & $1(2.8 \%)$ & $0(0.0 \%)$ & 0.250 \\
\hline Statin & $16(5.5 \%)$ & $6(16.7 \%)$ & $10(3.9 \%)$ & 0.006 \\
\hline Number of drugs acting on the ACE2 receptor ${ }^{+}$ & & & & $<0.001$ \\
\hline 0 & $266(90.8 \%)$ & $26(72.2 \%)$ & $240(93.4 \%)$ & \\
\hline 1 & $22(7.5 \%)$ & $6(16.7 \%)$ & $16(6.2 \%)$ & \\
\hline 2 & $4(1.4 \%)$ & $3(8.3 \%)$ & $1(0.4 \%)$ & \\
\hline 3 & $1(0.3 \%)$ & $1(2.8 \%)$ & $0(0.0 \%)$ & \\
\hline $\begin{array}{l}\text { Number of drugs acting on the ACE2 and/or } \\
\text { DDP } 4 \ddagger\end{array}$ & & & & $<0.001$ \\
\hline 0 & $249(85.0 \%)$ & $22(61.1 \%)$ & $227(88.3 \%)$ & \\
\hline 1 & $36(12.3 \%)$ & $9(25.0 \%)$ & $27(10.5 \%)$ & \\
\hline 2 & $6(2.0 \%)$ & $3(8.3 \%)$ & $3(1.2 \%)$ & \\
\hline 3 & $2(0.7 \%)$ & $2(5.6 \%)$ & $0(0.0 \%)$ & \\
\hline
\end{tabular}


Table 1. Cont.

\begin{tabular}{|c|c|c|c|c|c|}
\hline & & Total, No. (\%) & Progression, No. (\%) & $\begin{array}{c}\text { Improvement/ } \\
\text { Stabilization, No. (\%) }\end{array}$ & $p$ \\
\hline & & $(n=293)$ & $(n=36)$ & $(n=257)$ & Value \\
\hline \multicolumn{6}{|l|}{ Available laboratory findings, median (IQR) } \\
\hline WBC count $\left(10^{9} / \mathrm{L}\right)$ & $n=26$ & $6.3(5.1-8.5)$ & $6.0(5.2-7.7)$ & $8.5(5.8-9.0)$ & 0.364 \\
\hline Hemoglobin (g/dL) & $n=26$ & $13.9(12.6-14.9)$ & $14.0(12.9-14.9)$ & $13.7(11.8-15.1)$ & 0.644 \\
\hline Platelet count $\left(10^{9} / \mathrm{L}\right)$ & $n=26$ & $\begin{array}{c}198.5 \\
(156.0-295.0)\end{array}$ & $171.0(142.5-287.0)$ & $255.0(215.5-281.0)$ & 0.140 \\
\hline Neutrophil count $\left(10^{9} / \mathrm{L}\right)$ & $n=26$ & $4.6(3.0-6.3)$ & $4.6(3.2-5.3)$ & $6.0(3.4-6.8)$ & 0.885 \\
\hline Lymphocyte count $\left(10^{9} / \mathrm{L}\right)$ & $n=26$ & $1.4(1.1-1.7)$ & $1.4(1.0-1.7)$ & $1.5(1.3-2.1)$ & 0.364 \\
\hline Monocyte count $\left(10^{9} / \mathrm{L}\right)$ & $n=26$ & $0.4(0.3-0.7)$ & $0.4(0.3-0.7)$ & $0.6(0.3-0.6)$ & 0.729 \\
\hline Eosinophil count $\left(10^{9} / \mathrm{L}\right)$ & $n=26$ & $0.7(0.0-0.9)$ & $0.3(0.0-0.8)$ & $1.1(0.8-1.8)$ & 0.004 \\
\hline Activated partial thromboplastin time (sec) & $n=21$ & $28.8(27.5-32.2)$ & $30.0(28.1-33.1)$ & $27.5(27.1-27.6)$ & 0.099 \\
\hline Prothrombin time (sec) & $n=21$ & $10.6(10.2-11.3)$ & $10.8(10.4-11.4)$ & $10.2(9.7-10.4)$ & 0.089 \\
\hline BUN (mg/dl) & $n=26$ & $10.8(9.7-14.6)$ & $11.2(10.1-14.5)$ & $10.2(8.6-12.8)$ & 0.544 \\
\hline Serum creatinine $(\mathrm{ng} / \mathrm{mL})$ & $n=26$ & $0.9(0.7-1.0)$ & $1.0(0.8-1.1)$ & $0.7(0.6-0.9)$ & 0.056 \\
\hline Total protein $(\mathrm{g} / \mathrm{dL})$ & $n=26$ & $6.9(6.5-7.6)$ & $6.8(6.3-7.3)$ & $7.5(6.8-7.8)$ & 0.165 \\
\hline Albumin (g/dL) & $n=26$ & $4.0(3.7-4.2)$ & $3.8(3.4-4.1)$ & $4.2(4.0-4.4)$ & 0.081 \\
\hline AST (IU/L) & $n=26$ & $29.5(23.0-69.0)$ & $30.0(25.5-77.0)$ & $24.0(21.0-38.0)$ & 0.148 \\
\hline ALT (IU/L) & $n=26$ & $27.5(17.0-52.0)$ & $30.0(20.5-60.5)$ & $17.0(17.0-43.0)$ & 0.469 \\
\hline Alkaline phosphatase (IU/L) & $n=26$ & $75.0(64.0-94.0)$ & $78.0(69.0-94.0)$ & $67.0(63.5-84.5)$ & 0.563 \\
\hline CRP (mg/L) & $n=26$ & $3.8(0.5-6.5)$ & $4.8(1.5-7.7)$ & $0.5(0.5-2.3)$ & 0.081 \\
\hline
\end{tabular}

* IQR, interquartile range, ECOG, Eastern Cooperative Oncology Group performance status; SpO2, pulse oximeter oxygen saturation; ACE, angiotensin converting enzyme; DDP4, dipeptidyl peptidase 4; BUN, blood urea nitrogen; AST, aspartate aminotransferase; ALT, alanine aminotransferase; CRP, C-reactive protein. ${ }^{\dagger}$ Includes nonsteroidal anti-inflammatory drug, angiotensin II receptor blockers, and thiazolidinediones. $\ddagger$ Includes nonsteroidal anti-inflammatory drug, angiotensin II receptor blockers, thiazolidinediones, and dipeptidyl peptidase-4 inhibitors.

\subsubsection{After PS Matching}

We conducted PS matching to adjust baseline demographics and clinical variables between the progression and improvement/stabilization groups, resulting in 36 matched pairs of patients. Confounding variables were well balanced in the two groups, including all the 10 variables identified above in the Methods section (Table 2). After PS matching, prior history of drug use, including ibuprofen, ARB, DPP4i, was not statistically different between patients in the progression and improvement/stabilization groups. Similarly, the effect of these drugs on patient prognosis did not differ significantly in subgroup analysis of patients with hypertension (Table A2) or diabetes mellitus (Table A3).

Table 2. Baseline characteristics of patients with COVID-19, after propensity-score matching *

\begin{tabular}{llll}
\hline & Progression, No. (\%) & $\begin{array}{l}\text { Improvement/ } \\
\text { Stabilization, No. (\%) }\end{array}$ & $p$ \\
\hline Age, median (IQR) & $(\boldsymbol{n}=\mathbf{3 6 )}$ & $(\boldsymbol{n}=\mathbf{2 5 7 )}$ & Value \\
\hline Male sex & $49.5(34.0-57.0)$ & $45.5(27.5-54.5)$ & 0.185 \\
\hline Healthcare-associated infection & $24(66.7 \%)$ & $19(52.8 \%)$ & 0.336 \\
\hline ECOG performance status & $3(8.3 \%)$ & $2(5.6 \%)$ & $>0.999$ \\
\hline 0 & & & 0.109 \\
\hline 1 & $28(77.8 \%)$ & $31(86.1 \%)$ & \\
\hline 2 & $8(22.2 \%)$ & $3(8.3 \%)$ & \\
\hline
\end{tabular}


Table 2. Cont.

\begin{tabular}{|c|c|c|c|}
\hline & Progression, No. (\%) & $\begin{array}{l}\text { Improvement/ } \\
\text { Stabilization, No. (\%) }\end{array}$ & $p$ \\
\hline & $(n=36)$ & $(n=257)$ & Value \\
\hline $\begin{array}{l}\text { Time from disease confirmation to } \\
\text { admission, median (IQR), days }\end{array}$ & $4.0(3.0-6.0)$ & $5.0(3.0-8.0)$ & 0.222 \\
\hline $\begin{array}{l}\text { Time from symptom onset to admission, } \\
\text { median (IQR), days }\end{array}$ & $8.0(3.0-10.5)$ & $10.0(5.0-14.0)$ & 0.194 \\
\hline $\begin{array}{l}\text { Time from symptom onset to confirmation, } \\
\text { median (IQR), days }\end{array}$ & $3.0(0.0-6.0)$ & $3.0(0.5-8.0)$ & 0.740 \\
\hline \multicolumn{4}{|l|}{ Initial symptoms (may be multiple) } \\
\hline Asymptomatic & $3(8.3 \%)$ & $3(8.3 \%)$ & $>0.999$ \\
\hline Productive cough & $15(41.7 \%)$ & $14(38.9 \%)$ & $>0.999$ \\
\hline Fever & $20(55.6 \%)$ & $13(36.1 \%)$ & 0.156 \\
\hline Cough & $4(11.1 \%)$ & $9(25.0 \%)$ & 0.220 \\
\hline Headache & $16(44.4 \%)$ & $10(27.8 \%)$ & 0.220 \\
\hline Myalgia or fatigue & $14(38.9 \%)$ & $12(33.3 \%)$ & 0.806 \\
\hline Chills & $19(52.8 \%)$ & $9(25.0 \%)$ & 0.030 \\
\hline Sore throat & $7(19.4 \%)$ & $10(27.8 \%)$ & 0.579 \\
\hline Rhinorrhea & $4(11.1 \%)$ & $6(16.7 \%)$ & 0.733 \\
\hline Dyspnea & $10(27.8 \%)$ & $4(11.1 \%)$ & 0.137 \\
\hline Diarrhea & $7(19.4 \%)$ & $3(8.3 \%)$ & 0.307 \\
\hline Nausea or vomiting & $3(8.3 \%)$ & $3(8.3 \%)$ & $>0.999$ \\
\hline Chest pain & $4(11.1 \%)$ & $1(2.8 \%)$ & 0.354 \\
\hline \multicolumn{4}{|l|}{ Initial signs (may be multiple) } \\
\hline Body temperature, median (IQR), ${ }^{\circ} \mathrm{C}$ & $37.3(36.5-37.7)$ & $37.1(36.9-37.3)$ & 0.443 \\
\hline $\begin{array}{l}\text { Systolic blood pressure, median (IQR), } \\
\mathrm{mm} \mathrm{Hg}\end{array}$ & $135.5(121.5-146.0)$ & $136.5(125.5-142.0)$ & 0.897 \\
\hline $\begin{array}{l}\text { Diastolic blood pressure, median (IQR), } \\
\mathrm{mm} \mathrm{Hg}\end{array}$ & $82.0(75.5-90.5)$ & $82.5(74.0-89.0)$ & 0.778 \\
\hline Pulse rate, median (IQR), beats/min & $90.0(79.0-102.5)$ & $88.0(82.0-97.5)$ & 0.389 \\
\hline Respiratory rate, median (IQR), beats/min & $16.0(16.0-18.0)$ & $16.0(16.0-17.0)$ & 0.108 \\
\hline SpO2, median (IQR), \% & $98.0(97.0-99.0)$ & $98.0(98.0-99.0)$ & 0.438 \\
\hline \multicolumn{4}{|l|}{ Comorbidities (may be multiple) } \\
\hline Hypertension & $9(25.0 \%)$ & $8(22.2 \%)$ & $>0.999$ \\
\hline Diabetes mellitus & $10(27.8 \%)$ & $6(16.7 \%)$ & 0.395 \\
\hline Allergic diseases & $1(2.8 \%)$ & $3(8.3 \%)$ & 0.607 \\
\hline Chronic lung disease & $3(8.3 \%)$ & $3(8.3 \%)$ & $>0.999$ \\
\hline Peripheral vascular disease & $1(2.8 \%)$ & $5(13.9 \%)$ & 0.201 \\
\hline Malignant tumor(s) & $0(0.0 \%)$ & $0(0.0 \%)$ & NA \\
\hline Liver disease & $1(2.8 \%)$ & $1(2.8 \%)$ & $>0.999$ \\
\hline Cerebrovascular disease & $2(5.6 \%)$ & $1(2.8 \%)$ & $>0.999$ \\
\hline
\end{tabular}


Table 2. Cont.

\begin{tabular}{|c|c|c|c|}
\hline & Progression, No. $(\%)$ & $\begin{array}{l}\text { Improvement/ } \\
\text { Stabilization, No. (\%) }\end{array}$ & $p$ \\
\hline & $(n=36)$ & $(n=257)$ & Value \\
\hline Rheumatic disease & $1(2.8 \%)$ & $0(0.0 \%)$ & $>0.999$ \\
\hline Acute myocardial infarction & $0(0.0 \%)$ & $0(0.0 \%)$ & NA \\
\hline Congestive heart failure & $2(5.6 \%)$ & $0(0.0 \%)$ & 0.473 \\
\hline Kidney disease & $0(0.0 \%)$ & $0(0.0 \%)$ & NA \\
\hline \multicolumn{4}{|l|}{ Prior history of drug use } \\
\hline Ibuprofen & $6(16.7 \%)$ & $2(5.6 \%)$ & 0.261 \\
\hline Angiotensin II receptor blockers & $6(16.7 \%)$ & $4(11.1 \%)$ & 0.733 \\
\hline Calcium channel blocker & $5(13.9 \%)$ & $4(11.1 \%)$ & $>0.999$ \\
\hline Beta blocker & $2(5.6 \%)$ & $2(5.6 \%)$ & $>0.999$ \\
\hline Diuretic & $0(0.0 \%)$ & $1(2.8 \%)$ & $>0.999$ \\
\hline Dipeptidyl peptidase-4 inhibitor & $8(22.2 \%)$ & $5(13.9 \%)$ & 0.540 \\
\hline Metformin & $6(16.7 \%)$ & $3(8.3 \%)$ & 0.476 \\
\hline Sulfonylurea & $2(5.6 \%)$ & $1(2.8 \%)$ & $>0.999$ \\
\hline Thiazolidinedione & $1(2.8 \%)$ & $0(0.0 \%)$ & $>0.999$ \\
\hline SGLT2 inhibitor & $0(0.0 \%)$ & $0(0.0 \%)$ & NA \\
\hline Gabapentinoid & $30(83.3 \%)$ & $32(88.9 \%)$ & $>0.999$ \\
\hline Isosorbide & $1(2.8 \%)$ & $0(0.0 \%)$ & $>0.999$ \\
\hline Statin & $6(16.7 \%)$ & $3(8.3 \%)$ & 0.476 \\
\hline $\begin{array}{l}\text { Number of drugs acting on the ACE2 } \\
\text { receptor }{ }^{+}\end{array}$ & & & 0.542 \\
\hline 0 & $26(72.2 \%)$ & $28(77.8 \%)$ & \\
\hline 1 & $6(16.7 \%)$ & $7(19.4 \%)$ & \\
\hline 2 & $3(8.3 \%)$ & $1(2.8 \%)$ & \\
\hline 3 & $1(2.8 \%)$ & $0(0.0 \%)$ & \\
\hline $\begin{array}{l}\text { Number of drugs acting on the ACE2 } \\
\text { and/or DDP4 } \ddagger\end{array}$ & & & 0.343 \\
\hline 0 & $22(61.1 \%)$ & $26(72.2 \%)$ & \\
\hline 1 & $9(25.0 \%)$ & $9(25.0 \%)$ & \\
\hline 2 & $3(8.3 \%)$ & $1(2.8 \%)$ & \\
\hline 3 & $2(5.6 \%)$ & $0(0.0 \%)$ & \\
\hline
\end{tabular}

* IQR, interquartile range, ECOG, Eastern Cooperative Oncology Group performance status; SpO2, Pulse Oximeter Oxygen Saturation; ACE, angiotensin converting enzyme; DDP4, dipeptidyl peptidase $4 .{ }^{\dagger}$ Includes ibuprofen, angiotensin II receptor blockers, and thiazolidinediones. ‡ Includes ibuprofen, angiotensin II receptor blockers, thiazolidinediones, and dipeptidyl peptidase- 4 inhibitors.

\subsection{Comparison of the Predictive Models}

To confirm that KCDC classifications were suitable for initial triage of patients with COVID-19, the predictive values were compared to those of existing models using receiver operating characteristics analysis. As summarized in Table 3, all predictive values were significantly greater in the progression group than in the improvement/stabilization group $(p<0.001)$. KCDC classification I had the largest area under the curve (AUC, 0.817; 95\% CI, 0.740-0.895). After incorporating the CT score measured using baseline chest CT imaging into the KCDC classification I scheme, the AUC was $0.846(95 \% \mathrm{CI}$, 0.768-0.923), improving the predictive power. 
Table 3. Predictive models for disease severity and progression of patients with COVID-19*

\begin{tabular}{|c|c|c|c|c|c|c|c|c|}
\hline & Total, No. (\%) & $\begin{array}{l}\text { Progression, } \\
\text { No. }(\%)\end{array}$ & $\begin{array}{l}\text { Improvement/ } \\
\text { Stabilization, No. (\%) }\end{array}$ & $p$ & & & & \\
\hline & $(n=293)$ & $(n=36)$ & $(n=257)$ & Value & AUC & $95 \%$ CI & Sensitivity & Specificity \\
\hline KCDC classification I & & & & $<0.001$ & 0.817 & $(0.740-0.895)$ & $83.30 \%$ & $67.70 \%$ \\
\hline Class I & $180(61.4 \%)$ & $6(16.7 \%)$ & $174(67.7 \%)$ & & & & & \\
\hline Class II & $91(31.1 \%)$ & $14(38.9 \%)$ & $77(30.0 \%)$ & & & & & \\
\hline Class III & $22(7.5 \%)$ & $16(44.4 \%)$ & $6(2.3 \%)$ & & & & & \\
\hline KCDC classification II & & & & $<0.001$ & 0.676 & $(0.590-0.762)$ & $52.80 \%$ & $82.50 \%$ \\
\hline Class I & $229(78.2 \%)$ & $17(47.2 \%)$ & $212(82.5 \%)$ & & & & & \\
\hline Class III & $64(21.8 \%)$ & $19(52.8 \%)$ & $45(17.5 \%)$ & & & & & \\
\hline CT score, median (IQR) & $0.0(0.0-4.0)$ & $6.2(1.0-14.5)$ & $0.0(0.0-3.0)$ & $<0.001$ & 0.768 & $(0.680-0.856)$ & $77.80 \%$ & $65.00 \%$ \\
\hline MuLBSTA, median (IQR) & $0.0(0.0-5.0)$ & $5.0(5.0-7.0)$ & $0.0(0.0-5.0)$ & $<0.001$ & 0.744 & $(0.662-0.825)$ & $77.80 \%$ & $63.80 \%$ \\
\hline CURB65 & & & & $<0.001$ & 0.575 & $(0.507-0.642)$ & $19.40 \%$ & $95.30 \%$ \\
\hline 0 & $274(93.5 \%)$ & $29(80.6 \%)$ & $245(95.3 \%)$ & & & & & \\
\hline 1 & $18(6.1 \%)$ & $6(16.7 \%)$ & $12(4.7 \%)$ & & & & & \\
\hline 2 & $1(0.3 \%)$ & $1(2.8 \%)$ & $0(0.0 \%)$ & & & & & \\
\hline Pneumonia severity index & & & & $<0.001$ & 0.659 & $(0.568-0.750)$ & $50.00 \%$ & $79.00 \%$ \\
\hline Class I & $221(75.4 \%)$ & $18(50.0 \%)$ & $203(79.0 \%)$ & & & & & \\
\hline Class II & $53(18.1 \%)$ & $10(27.8 \%)$ & $43(16.7 \%)$ & & & & & \\
\hline Class III & $17(5.8 \%)$ & $6(16.7 \%)$ & $11(4.3 \%)$ & & & & & \\
\hline Class IV & $2(0.7 \%)$ & $2(5.6 \%)$ & $0(0.0 \%)$ & & & & & \\
\hline $\begin{array}{l}\text { Age-adjusted Charlson } \\
\text { comorbidity index, median (IQR) }\end{array}$ & $0.0(0.0-1.0)$ & $1.0(0.5-4.0)$ & $0.0(0.0-1.0)$ & $<0.001$ & 0.703 & $(0.610-0.795)$ & $75.00 \%$ & $56.80 \%$ \\
\hline
\end{tabular}

* AUC, area under the curve; CI, confidence interval; KCDC, Korea Centers for Disease Control and Prevention; CT, computed tomography, IQR, interquartile range; MuLBSTA, Multilobular infiltration, hypo-Lymphocytosis, Bacterial coinfection, Smoking history, hyper-Tension and Age; CURB65, Confusion, Urea, Respiratory rate, Blood pressure plus age $\geq$ 65 years. 


\subsection{Lopinavir/Ritonavir Treatment Outcomes}

Of the 293 patients with COVID-19, 30 were treated with lopinavir/ritonavir (Table A4). Patients chosen to receive lopinavir/ritonavir treatment were more likely to be in a higher risk group than patients who did not receive lopinavir/ritonavir treatment. After adjusting for confounding variables via PS matching, there were no significant differences between the groups for any of the 10 characteristics identified in the Methods section above. However, even after matching, 18 of $30(60.0 \%)$ patients who received lopinavir/ritonavir treatment showed disease progression, while 6 of $30(20.0 \%)$ patients who did not receive lopinavir/ritonavir treatment experienced disease progression. Patients treated in the lopinavir/ritonavir group had significantly shorter PFS than that in the group not receiving lopinavir/ritonavir both before and after PS matching, but there was no significant difference in the proportion of discharged patients between the two groups (Figure 2 and Table A5).

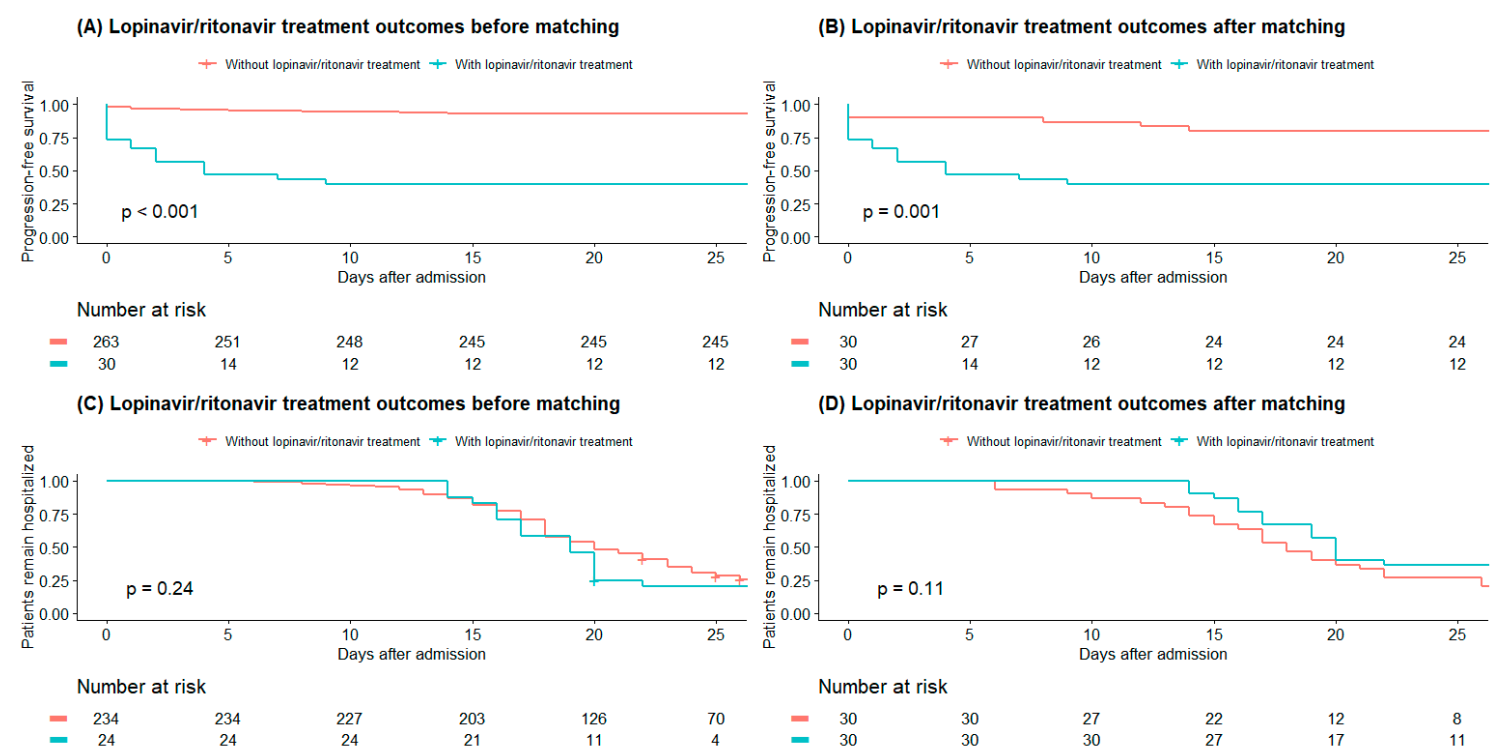

Figure 2. Kaplan-Meier survival analysis of progression-free survival $(\mathbf{A}, \mathbf{B})$ and proportion of patients requiring hospitalization (C,D) according to lopinavir/ritonavir treatment for patients with COVID-19 before and after propensity-score matching Figure legend: Patients treated with lopinavir/ritonavir group showed significantly lower progression-free survival than the without lopinavir/ritonavir group before and after propensity-score matching. but there was no statistical difference in discharge proportion between the two groups.

\section{Discussions}

In this cohort study, we reported the clinical characteristics of COVID-19 patients and risk factors associated with disease progression, especially those associated with early stages of the disease. We also assessed the usefulness of the KCDC classification for initial patient triage.

There are considerable differences between our study and previous studies of the course and severity of COVID-19. In previous reports addressing Chinese COVID-19 patients, most of the patients were middle-aged and elderly, presented fever and/or cough, and chest CT indicated pneumonia in most patients $[4,6,7,12,13,26,27]$. In our study, the median age was 29 years, $33.1 \%$ of patients were asymptomatic at hospital admission, and pneumonia was evident in chest CT scans in only $41.3 \%$ of patients. In South Korea, COVID-19 patients were identified and classified at an early stage through use of large-scale diagnostic testing in accordance with national policy, which allowed both asymptomatic and symptomatic patients to receive inpatient treatment.

The risk factors significantly associated with disease progression were older age, healthcareassociated infection, ECOG performance status, presence of initial symptoms at the time of hospital admission, higher initial PR, lower initial SpO2, hypertension, and diabetes, which were consistent 
with prior reports $[4,6,12,21,23,28]$. In contrast, asymptomatic cases at the time of hospital admission had favorable outcomes.

SARS-CoV-2 infects host cells by interacting with the angiotensin-converting enzyme (ACE) 2 receptors [29], which are expressed by epithelial cells in the lung, kidney, intestine, and blood vessels [30]. The high prevalence of ARDS and gastrointestinal symptoms such as diarrhea, nausea, and vomiting can be explained by this ACE-2-receptor-mediated mechanism in COVID-19 patients [31]. ACE inhibitors (ACEi) and ARBs that affect the renin-angiotensin-aldosterone system (RAAS) are commonly recommended for patients with hypertension [32]. In an animal model [33] and human studies [34,35], administration of ACEi and ARBs has been shown to increase the number of ACE2 receptors; ibuprofen and thiazolidinediones may also increase ACE2 expression [36]. Therefore, prior use of these drugs may be a risk factor for SARS-CoV 2 infection. Similar to the ACE2 receptor, human CD26 (also called DPP4) is also suggested as the potential binding site for COVID-19 [37]. Thus, DPP4i, which is widely used as a diabetes drug, may produce effects similar to ARBs in COVID-19 patients. However, given only small-scale clinical studies addressing ACEi/ARB use and patient outcomes in hospital settings [28] have been completed to date, the impact of these drugs on COVID-19 is controversial. Moreover, Vaduganathan et al. suggested that recombinant ACE2 protein may restore balance to the RAAS and potentially prevent organ damage, and drugs acting on ACE2 may benefit rather than harm COVID-19 patients [38]. To analyze the impact of drugs acting on the ACE2 receptor and human CD26 in COVID-19 patients, we conducted a PS matched study. Before matching, the proportion of patients reporting prior use of these drugs was significantly greater in the progression group; however, after adjusting for 10 confounding variables, including underlying comorbidities, there was no significant difference between patients with and without these medication histories. Even after comparing the sums of three (ibuprofen, ARBs, and thiazolidinediones) or four drugs (ibuprofen, ARBs, thiazolidinediones, and DPP4i), no significant differences were found between the two groups. Furthermore, in subgroup analysis of patients with hypertension and diabetes mellitus, the effect of these drugs on patient prognosis was not statistically significant. These results suggest that, in diseases such as hypertension and diabetes mellitus, the underlying pathophysiology associated with the RAAS affects the prognosis of COVID-19 patients rather than the pharmacologic effects of the drugs used to control the disease.

In the global COVID-19 pandemic, the major challenge is the lack of medical resources. We evaluated use of the KCDC classifications to triage patients with COVID-19 according to severity of the disease and to ensure they are treated at the appropriate medical institution. Our results indicated that KCDC classification I had a good AUC $(0.817 ; 95 \%$ CI 73.98-89.46) and sensitivity, which suggested that this model is suitable for early screening of low-risk patients who are less likely to progress to severe disease. The use of the triage algorithm and KCDC classification for COVID-19 patients saves medical resources, allowing more efficient treatment and management of patients. Using the KCDC classification as a predictive model in the early stages of COVID-19 outbreaks, more medical resources could be focused on patients with more severe disease, which may have underlain the relatively low CFR in South Korea.

The CT scores (AUC > 0.7) for the COVID-19 patients in this study clearly distinguished the progression group from the improvement/stabilization group, a finding which is consistent with previous reports [21-23]. The use of the KCDC classification I scheme with the CT score increased the AUC and specificity of the predictive model. Therefore, we suggest that triaging patients by applying these predictive models in accordance with the medical conditions and policies of each country may help manage patients in the COVID-19 pandemic situation.

In our clinical study, which comprised mostly mild to moderate cases, patients who received lopinavir/ritonavir treatment were not likely to experience a decrease in PFS; rather, the patients' symptoms may have been aggravated due to side effects of the antivirals. In a previous randomized controlled trial conducted in patients with severe COVID-19, there was no treatment benefit of 
lopinavir/ritonavir: of 95 patients receiving lopinavir/ritonavir treatment, $48(48.4 \%)$ had gastrointestinal side effects [39], which is consistent with our data.

The retrospective and single-center nature of our study may limit wider applicability of the results. Due to the limited number of cases in the progression group, it was difficult to analyze risk factors for disease progression using multivariable-adjusted methods. Thus, hidden bias and residual confounding factors might have influenced our results. Another limitation of our study was that, to protect medical staff and minimize further spread of the disease in the hospital setting, routine laboratory tests were not conducted in all patients, and these data were not available for inclusion in the analyses. However, we tried to analyze risk factors for disease progression and treatment outcomes for COVID-19 patients while minimizing selection bias using the PS matched study.

After controlling for potential biases using PS matching analysis, drugs acting on the ACE2 receptor and human CD26 were not risk factors for disease progression. We also demonstrated that the KCDC classification I was able to distinguish the improvement/stabilization group from the progression group of COVID-19 patients, and the triage algorithm system saved medical resources, enabling efficient treatment and management of COVID-19 patients in South Korea.

Author Contributions: Conceptualization, M.H.C., H.A., and S.H.J.; data curation, M.H.C. and H.A.; writingoriginal draft, M.H.C.; writing-review and editing, H.S.R., B.-J.K., J.J., M.J., and J.K. All authors have read and agreed to the published version of the manuscript.

Conflicts of Interest: The authors declare no conflicts of interest.

\section{Appendix A}

Table A1. Logistic regression of risk factors for COVID-19 disease progression *.

\begin{tabular}{llll}
\hline & OR & $\mathbf{9 5 \%}$ CI & $p$ \\
\hline Age & 1.07 & $1.04-1.10$ & $<0.001$ \\
Male sex & 0.71 & $0.33-1.49$ & 0.360 \\
Healthcare-associated infection & 7.70 & $1.49-39.71$ & 0.015 \\
ECOG performance status & 5.68 & $2.07-15.56$ & 0.001 \\
Time from disease confirmation to admission, days & 0.97 & $0.86-1.09$ & 0.567 \\
Time from symptom onset to admission, days & 1.02 & $0.98-1.07$ & 0.312 \\
Time from symptom onset to confirmation, days & 1.03 & $0.97-1.09$ & 0.322 \\
Time from admission to discharge, days & 0.95 & $0.87-1.04$ & 0.304 \\
Symptom duration, days & 1.07 & $1.04-1.11$ & 0.001 \\
\hline Initial symptoms & & & \\
Asymptomatic & 0.16 & $0.05-0.53$ & 0.003 \\
Productive cough & 1.99 & $0.97-4.07$ & 0.061 \\
Fever & 4.59 & $2.23-9.45$ & $<0.001$ \\
Cough & 0.37 & $0.13-1.08$ & 0.070 \\
Headache & 3.77 & $1.81-7.84$ & $<0.001$ \\
Myalgia or fatigue & 2.92 & $1.39-6.13$ & 0.005 \\
Chills & 7.09 & $3.37-14.93$ & $<0.001$ \\
Sore throat & 1.53 & $0.62-3.76$ & 0.353 \\
Rhinorrhea & 0.88 & $0.29-2.65$ & 0.819 \\
Dyspnea & 7.85 & $3.09-19.93$ & $<0.001$ \\
Diarrhea & 4.93 & $1.80-13.51$ & 0.002 \\
Nausea or vomiting & 3.80 & $0.91-15.93$ & 0.068 \\
Chest pain & 6.30 & $1.61-24.68$ & 0.008 \\
\hline
\end{tabular}


Table A1. Cont.

\begin{tabular}{|c|c|c|c|}
\hline & OR & $95 \% \mathrm{CI}$ & $p$ \\
\hline \multicolumn{4}{|l|}{ Initial signs } \\
\hline Body temperature, ${ }^{\circ} \mathrm{C}$ & 9.41 & $3.98-22.25$ & $<0.001$ \\
\hline Systolic blood pressure, $\mathrm{mm} \mathrm{Hg}$ & 1.01 & $0.99-1.03$ & 0.402 \\
\hline Diastolic blood pressure, $\mathrm{mm} \mathrm{Hg}$ & 1.03 & $1.00-1.06$ & 0.048 \\
\hline Pulse rate, beats/min & 1.04 & $1.01-1.06$ & 0.010 \\
\hline Respiratory rate, breaths/min & 1.12 & $0.94-1.33$ & 0.218 \\
\hline $\mathrm{SpO} 2, \%$ & 0.71 & $0.55-0.91$ & 0.007 \\
\hline \multicolumn{4}{|l|}{ Comorbidities } \\
\hline Hypertension & 3.95 & $1.64-9.54$ & 0.002 \\
\hline Diabetes mellitus & 8.60 & $3.34-22.17$ & $<0.001$ \\
\hline Allergic disease & 0.17 & $0.02-1.28$ & 0.085 \\
\hline Chronic lung disease & 1.58 & $0.43-5.78$ & 0.491 \\
\hline Peripheral vascular disease & 0.58 & $0.07-4.63$ & 0.610 \\
\hline Malignant tumors & NA & & \\
\hline Liver disease & 1.81 & $0.20-16.63$ & 0.601 \\
\hline Congestive heart failure & 3.72 & $0.66-21.09$ & 0.138 \\
\hline Cerebrovascular disease & 4.98 & $0.80-30.88$ & 0.085 \\
\hline Rheumatic disease & 7.31 & $0.45-119.59$ & 0.163 \\
\hline Acute myocardial infarction & NA & & \\
\hline Kidney disease & NA & & \\
\hline \multicolumn{4}{|l|}{ Prior history of drug use } \\
\hline Ibuprofen & 3.23 & $1.16-8.95$ & 0.024 \\
\hline Angiotensin II receptor blocker & 4.94 & $1.68-14.56$ & 0.004 \\
\hline Calcium channel blocker & 3.61 & $1.18-11.07$ & 0.025 \\
\hline Beta blocker & 2.10 & $0.42-10.53$ & 0.367 \\
\hline Diuretic & NA & & \\
\hline Dipeptidyl peptidase- 4 inhibitor & 8.89 & $3.10-25.54$ & $<0.001$ \\
\hline Metformin & 8.37 & $2.54-27.59$ & 0.001 \\
\hline Sulfonylurea & 7.50 & $1.02-1.55$ & 0.048 \\
\hline Thiazolidinedione & NA & & \\
\hline Sodium-glucose cotransporter-2 inhibitors & NA & & \\
\hline Gabapentinoid & NA & & \\
\hline Isosorbide & NA & & \\
\hline Statin & 4.94 & $1.68-14.56$ & 0.004 \\
\hline Number of drugs acting on the ACE2 receptor ${ }^{+}$ & 4.37 & $2.14-8.92$ & $<0.001$ \\
\hline Number of drugs acting on the ACE2 and/or DDP4 $\ddagger$ & 3.66 & $2.05-6.55$ & $<0.001$ \\
\hline KCDC classification I & 8.67 & $4.57-16.45$ & $<0.001$ \\
\hline KCDC classification II & 2.29 & $1.59-3.30$ & $<0.001$ \\
\hline CT score & 1.22 & $1.15-1.31$ & $<0.001$ \\
\hline MuLBSTA & 1.28 & $1.16-1.42$ & $<0.001$ \\
\hline CURB65 & 4.86 & $1.88-12.57$ & 0.001 \\
\hline Pneumonia severity index & 2.81 & $1.76-4.47$ & $<0.001$ \\
\hline Age-adjusted Charlson comorbidity index & 1.73 & $1.36-2.20$ & $<0.001$ \\
\hline
\end{tabular}

* OR, odds ratio; CI, confidence interval; NA, not available; ECOG, Eastern Cooperative Oncology Group performance status; SpO2, Pulse Oximeter Oxygen Saturation; KCDC, Korea Centers for Disease Control and Prevention; CT, computed tomography; MuLBSTA, Multilobular infiltration, hypo-Lymphocytosis, Bacterial coinfection, Smoking history, hyper-Tension and Age; CURB65, Confusion, Urea, Respiratory rate, Blood pressure plus age $\geq 65$ years. ${ }^{\dagger}$ Includes ibuprofen, angiotensin II receptor blockers, and thiazolidinediones. ${ }^{\ddagger}$ Includes ibuprofen, angiotensin II receptor blockers, thiazolidinediones, and dipeptidyl peptidase-4 inhibitor. 
Table A2. Subgroup analysis of the effect of prior use of drugs in COVID-19 patients with hypertension.

\begin{tabular}{llll}
\hline & Progression, No. (\%) & $\begin{array}{l}\text { Improvement/ } \\
\text { Stabilization, No. (\%) }\end{array}$ & $p$ \\
\hline & $(\boldsymbol{n}=\mathbf{9 )}$ & $(\boldsymbol{n = 2 0 )}$ & Value \\
\hline Age, median (IQR) & $55(53.0-61.0)$ & $51.5(48.0-55.5)$ & 0.125 \\
Angiotensin II receptor blocker & $6(66.7 \%)$ & $10(50.0 \%)$ & 0.666 \\
Calcium channel blocker & $5(55.6 \%)$ & $11(55.0 \%)$ & $>0.999$ \\
Ibuprofen & $2(22.2 \%)$ & $1(5.0 \%)$ & 0.453 \\
Beta blocker & $2(22.2 \%)$ & $2(10.0 \%)$ & 0.763 \\
Diuretic & $0(0.0 \%)$ & $2(10.0 \%)$ & 0.848 \\
\hline
\end{tabular}

Table A3. Subgroup analysis of the effect of prior use of drugs in COVID-19 patients with diabetes mellitus.

\begin{tabular}{llll}
\hline & Progression, No. (\%) & $\begin{array}{l}\text { Improvement/ } \\
\text { Stabilization, No. (\%) }\end{array}$ & $p$ \\
\hline & $(\boldsymbol{n}=\mathbf{1 0})$ & $(\boldsymbol{n}=\mathbf{1 1})$ & Value \\
\hline Age, median (IQR) & $52.5(47.0-55.0)$ & $52.0(51.0-55.0)$ & 0.915 \\
\hline Dipeptidyl peptidase-4 inhibitor & $8(80.0 \%)$ & $8(72.7 \%)$ & $>0.999$ \\
\hline Metformin & $6(60.0 \%)$ & $6(54.5 \%)$ & $>0.999$ \\
\hline Angiotensin II receptor blocker & $4(40.0 \%)$ & $1(9.1 \%)$ & 0.251 \\
\hline Ibuprofen & $1(10.0 \%)$ & $1(9.1 \%)$ & $>0.999$ \\
\hline Thiazolidinedione & $1(10.0 \%)$ & $0(0.0 \%)$ & 0.476 \\
\hline Sulfonylurea & $2(20.0 \%)$ & $2(18.2 \%)$ & $>0.999$ \\
\hline Sodium-glucose cotransporter-2 inhibitor & $0(0.0 \%)$ & $1(9.1 \%)$ & $>0.999$ \\
\hline Gabapentinoid & $0(0.0 \%)$ & $1(9.1 \%)$ & $>0.999$ \\
\hline Isosorbide & $1(10.0 \%)$ & $0(0.0 \%)$ & 0.476 \\
\hline
\end{tabular}

Table A4. Lopinavir/ritonavir treatment outcomes in COVID-19 patients before and after propensity-score matching *.

\begin{tabular}{|c|c|c|c|c|c|c|}
\hline & \multicolumn{3}{|c|}{ Before Propensity-Score Matching } & \multicolumn{3}{|c|}{ After Propensity-Score Matching } \\
\hline & $\begin{array}{l}\text { With Lopinavir/ } \\
\text { Ritonavir } \\
\text { Treatment }\end{array}$ & $\begin{array}{l}\text { Without } \\
\text { Lopinavir/ } \\
\text { Ritonavir } \\
\text { Treatment }\end{array}$ & $p$ & $\begin{array}{l}\text { With Lopinavir/ } \\
\text { Ritonavir } \\
\text { Treatment }\end{array}$ & $\begin{array}{l}\text { Without } \\
\text { Lopinavir/ } \\
\text { Ritonavir } \\
\text { Treatment }\end{array}$ & $p$ \\
\hline & No. $(\%)(n=30)$ & No. $(\%)(n=263)$ & Value & No. $(\%)(n=30)$ & No. $(\%)(n=30)$ & Value \\
\hline Disease progression & $18(60.0 \%)$ & $18(6.8 \%)$ & $<0.001$ & $18(60.0 \%)$ & $6(20.0 \%)$ & 0.004 \\
\hline Age, median (IQR) & $54(47-59)$ & $27(23-46)$ & $<0.001$ & $54(47-59)$ & $51(46-55)$ & 0.230 \\
\hline Male sex & $19(63.3 \%)$ & $195(74.1 \%)$ & 0.295 & $19(63.3 \%)$ & $9(30.0 \%)$ & 0.020 \\
\hline Healthcare-associated infection & $4(13.3 \%)$ & $2(0.8 \%)$ & $<0.001$ & $4(13.3 \%)$ & $2(6.7 \%)$ & 0.667 \\
\hline ECOG performance status & & & $<0.001$ & & & 0.041 \\
\hline 0 & $19(63.3 \%)$ & $260(98.9 \%)$ & & $19(63.3 \%)$ & $27(90.0 \%)$ & \\
\hline 1 & $9(30.0 \%)$ & $3(1.1 \%)$ & & $9(30.0 \%)$ & $3(10.0 \%)$ & \\
\hline 2 & $2(6.7 \%)$ & $0(0.0 \%)$ & & $2(6.7 \%)$ & $0(0.0 \%)$ & \\
\hline $\begin{array}{l}\text { Time from disease confirmation to } \\
\text { admission, median (IQR), days }\end{array}$ & $3.5(3.0-5.0)$ & $5.0(3.0-7.0)$ & 0.037 & $3.5(3.0-5.0)$ & $5.0(3.0-7.0)$ & 0.058 \\
\hline $\begin{array}{l}\text { Time from symptom onset to } \\
\text { admission, median (IQR), days }\end{array}$ & $6.0(4.0-9.0)$ & $6.0(0.0-12.0)$ & 0.336 & $6.0(4.0-9.0)$ & $11.0(8.0-15.0)$ & 0.001 \\
\hline $\begin{array}{l}\text { Time from symptom onset to } \\
\text { confirmation, median (IQR), days } \\
\text { Initial symptoms (may be multiple) }\end{array}$ & $3.0(0.0-5.0)$ & $1.0(0.0-6.0)$ & 0.283 & $3.0(0.0-5.0)$ & $5.0(2.0-9.0)$ & 0.032 \\
\hline
\end{tabular}


Table A4. Cont.

\begin{tabular}{|c|c|c|c|c|c|c|}
\hline & \multicolumn{3}{|c|}{ Before Propensity-Score Matching } & \multicolumn{3}{|c|}{ After Propensity-Score Matching } \\
\hline & $\begin{array}{l}\text { With Lopinavir/ } \\
\text { Ritonavir } \\
\text { Treatment }\end{array}$ & $\begin{array}{l}\text { Without } \\
\text { Lopinavir/ } \\
\text { Ritonavir } \\
\text { Treatment }\end{array}$ & $p$ & $\begin{array}{l}\text { With Lopinavir/ } \\
\text { Ritonavir } \\
\text { Treatment }\end{array}$ & $\begin{array}{l}\text { Without } \\
\text { Lopinavir/ } \\
\text { Ritonavir } \\
\text { Treatment }\end{array}$ & $p$ \\
\hline & No. $(\%)(n=30)$ & No. $(\%)(n=263)$ & Value & No. $(\%)(n=30)$ & No. $(\%)(n=30)$ & Value \\
\hline Asymptomatic & $0(0.0 \%)$ & $97(36.9 \%)$ & $<0.001$ & $0(0.0 \%)$ & $0(0.0 \%)$ & NA \\
\hline Productive cough & $12(40.0 \%)$ & $71(27.0 \%)$ & 0.199 & $12(40.0 \%)$ & $18(60.0 \%)$ & 0.197 \\
\hline Fever & $18(60.0 \%)$ & $57(21.7 \%)$ & $<0.001$ & $18(60.0 \%)$ & $16(53.3 \%)$ & 0.794 \\
\hline Cough & $6(20.0 \%)$ & $63(24.0 \%)$ & 0.798 & $6(20.0 \%)$ & $8(26.7 \%)$ & 0.760 \\
\hline Headache & $17(56.7 \%)$ & $44(16.7 \%)$ & $<0.001$ & $17(56.7 \%)$ & $9(30.0 \%)$ & 0.068 \\
\hline Myalgia or fatigue & $13(43.3 \%)$ & $47(17.9 \%)$ & 0.002 & $13(43.3 \%)$ & $7(23.3 \%)$ & 0.171 \\
\hline Chills & $16(53.3 \%)$ & $38(14.4 \%)$ & $<0.001$ & $16(53.3 \%)$ & $10(33.3 \%)$ & 0.193 \\
\hline Sore throat & $9(30.0 \%)$ & $33(12.5 \%)$ & 0.021 & $9(30.0 \%)$ & $7(23.3 \%)$ & 0.770 \\
\hline Rhinorrhea & $2(6.7 \%)$ & $34(12.9 \%)$ & 0.486 & $2(6.7 \%)$ & $2(6.7 \%)$ & $>0.999$ \\
\hline Dyspnea & $11(36.7 \%)$ & $11(4.2 \%)$ & $<0.001$ & $11(36.7 \%)$ & $4(13.3 \%)$ & 0.074 \\
\hline Diarrhea & $8(26.7 \%)$ & $11(4.2 \%)$ & $<0.001$ & $8(26.7 \%)$ & $1(3.3 \%)$ & 0.030 \\
\hline Nausea or vomiting & $4(13.3 \%)$ & $5(1.9 \%)$ & 0.004 & $4(13.3 \%)$ & $1(3.3 \%)$ & 0.350 \\
\hline Chest pain & $3(10.0 \%)$ & $6(2.3 \%)$ & 0.078 & $3(10.0 \%)$ & $2(6.7 \%)$ & $>0.999$ \\
\hline \multicolumn{7}{|l|}{ Initial signs (may be multiple) } \\
\hline $\begin{array}{l}\text { Body temperature, median } \\
(\mathrm{IQR}),{ }^{\circ} \mathrm{C}\end{array}$ & $37.2(36.5-37.5)$ & $36.7(36.5-37.0)$ & 0.010 & $37.2(36.5-37.5)$ & $37.0(36.6-37.3)$ & 0.784 \\
\hline $\begin{array}{l}\text { Systolic blood pressure, median } \\
\text { (IQR), } \mathrm{mm} \mathrm{Hg}\end{array}$ & $133.5(115.0-140.0)$ & $133.0(122.0-141.0)$ & 0.392 & $133.5(115.0-140.0)$ & $133.5(123.0-142.0)$ & 0.395 \\
\hline $\begin{array}{l}\text { Diastolic blood pressure, median } \\
\text { (IQR), } \mathrm{mm} \mathrm{Hg}\end{array}$ & $81.0(74.0-86.0)$ & $78.0(72.0-85.0)$ & 0.379 & $81.0(74.0-86.0)$ & $75.5(73.0-82.0)$ & 0.411 \\
\hline $\begin{array}{l}\text { Pulse rate, median (IQR), } \\
\text { beats/min }\end{array}$ & $87.0(77.0-98.0)$ & $86.0(75.5-95.5)$ & 0.340 & $87.0(77.0-98.0)$ & $89.0(80.0-100.0)$ & 0.700 \\
\hline $\begin{array}{l}\text { Respiratory rate, median (IQR), } \\
\text { beats/min }\end{array}$ & $17.0(16.0-18.0)$ & $16.0(16.0-18.0)$ & 0.098 & $17.0(16.0-18.0)$ & $16.0(16.0-20.0)$ & 0.666 \\
\hline SpO2, median (IQR), \% & $98.0(97.0-99.0)$ & $98.0(98.0-99.0)$ & 0.031 & $98.0(97.0-99.0)$ & $98.0(97.0-99.0)$ & 0.284 \\
\hline Comorbidities (may be multiple) & & & 0.000 & & & 0.000 \\
\hline Hypertension & $9(30.0 \%)$ & $20(7.6 \%)$ & $<0.001$ & $9(30.0 \%)$ & $7(23.3 \%)$ & 0.770 \\
\hline Diabetes mellitus & $8(26.7 \%)$ & $13(4.9 \%)$ & $<0.001$ & $8(26.7 \%)$ & $5(16.7 \%)$ & 0.531 \\
\hline Allergic disease & $0(0.0 \%)$ & $38(14.4 \%)$ & 0.052 & $0(0.0 \%)$ & $6(20.0 \%)$ & 0.031 \\
\hline Chronic lung disease & $2(6.7 \%)$ & $15(5.7 \%)$ & $>0.999$ & $2(6.7 \%)$ & $4(13.3 \%)$ & 0.667 \\
\hline Peripheral vascular disease & $1(3.3 \%)$ & $12(4.6 \%)$ & $>0.999$ & $1(3.3 \%)$ & $5(16.7 \%)$ & 0.197 \\
\hline Malignant tumors & $2(6.7 \%)$ & $5(1.9 \%)$ & 0.323 & $2(6.7 \%)$ & $2(6.7 \%)$ & $>0.999$ \\
\hline Liver disease & $0(0.0 \%)$ & $5(1.9 \%)$ & 0.986 & $0(0.0 \%)$ & $0(0.0 \%)$ & NA \\
\hline Congestive heart failure & $3(10.0 \%)$ & $3(1.1 \%)$ & 0.010 & $3(10.0 \%)$ & $0(0.0 \%)$ & 0.236 \\
\hline Cerebrovascular disease & $1(3.3 \%)$ & $4(1.5 \%)$ & $>0.999$ & $1(3.3 \%)$ & $2(6.7 \%)$ & $>0.999$ \\
\hline Rheumatic disease & $1(3.3 \%)$ & $1(0.4 \%)$ & 0.490 & $1(3.3 \%)$ & $0(0.0 \%)$ & $>0.999$ \\
\hline Acute myocardial infarction & $1(3.3 \%)$ & $0(0.0 \%)$ & 0.189 & $1(3.3 \%)$ & $0(0.0 \%)$ & $>0.999$ \\
\hline Kidney disease & $0(0.0 \%)$ & $1(0.4 \%)$ & $>0.999$ & $0(0.0 \%)$ & $0(0.0 \%)$ & NA \\
\hline \multicolumn{7}{|l|}{ Prior history of drug use } \\
\hline Ibuprofen & $6(20.0 \%)$ & $15(5.7 \%)$ & 0.012 & $6(20.0 \%)$ & $6(20.0 \%)$ & $>0.999$ \\
\hline Angiotensin II receptor blocker & $5(16.7 \%)$ & $11(4.2 \%)$ & 0.015 & $5(16.7 \%)$ & $4(13.3 \%)$ & $>0.999$ \\
\hline Calcium channel blocker & $6(20.0 \%)$ & $10(3.8 \%)$ & 0.001 & $6(20.0 \%)$ & $2(6.7 \%)$ & 0.255 \\
\hline Beta blocker & $3(10.0 \%)$ & $6(2.3 \%)$ & 0.078 & $3(10.0 \%)$ & $1(3.3 \%)$ & 0.605 \\
\hline Diuretic & $0(0.0 \%)$ & $2(0.8 \%)$ & $>0.999$ & $0(0.0 \%)$ & $1(3.3 \%)$ & $>0.999$ \\
\hline Dipeptidyl peptidase-4 inhibitor & $7(23.3 \%)$ & $9(3.4 \%)$ & $<0.001$ & $7(23.3 \%)$ & $5(16.7 \%)$ & 0.747 \\
\hline Metformin & $6(20.0 \%)$ & $6(2.3 \%)$ & $<0.001$ & $6(20.0 \%)$ & $3(10.0 \%)$ & 0.470 \\
\hline Sulfonylurea & $2(6.7 \%)$ & $2(0.8 \%)$ & 0.070 & $2(6.7 \%)$ & $1(3.3 \%)$ & $>0.999$ \\
\hline Thiazolidinediones & $0(0.0 \%)$ & $1(0.4 \%)$ & $>0.999$ & $0(0.0 \%)$ & $0(0.0 \%)$ & NA \\
\hline $\begin{array}{l}\text { Sodium-glucose cotransporter-2 } \\
\text { inhibitor }\end{array}$ & $0(0.0 \%)$ & $1(0.4 \%)$ & $>0.999$ & $0(0.0 \%)$ & $0(0.0 \%)$ & NA \\
\hline Gabapentinoid & $0(0.0 \%)$ & $1(0.4 \%)$ & $>0.999$ & $0(0.0 \%)$ & $0(0.0 \%)$ & NA \\
\hline Isosorbide & $1(3.3 \%)$ & $0(0.0 \%)$ & 0.189 & $1(3.3 \%)$ & $0(0.0 \%)$ & $>0.999$ \\
\hline Statin & $7(23.3 \%)$ & $9(3.4 \%)$ & $<0.001$ & $7(23.3 \%)$ & $4(13.3 \%)$ & 0.505 \\
\hline $\begin{array}{l}\text { Number of drugs acting on the } \\
\text { ACE2 receptor }\end{array}$ & & & $<0.001$ & & & 0.577 \\
\hline 0 & $21(70.0 \%)$ & $245(93.2 \%)$ & & $21(70.0 \%)$ & $22(73.3 \%)$ & \\
\hline 1 & $6(20.0 \%)$ & $16(6.1 \%)$ & & $6(20.0 \%)$ & $7(23.3 \%)$ & \\
\hline 2 & $3(10.0 \%)$ & $1(0.4 \%)$ & & $3(10.0 \%)$ & $1(3.3 \%)$ & \\
\hline 3 & $0(0.0 \%)$ & $1(0.4 \%)$ & & $0(0.0 \%)$ & $0(0.0 \%)$ & \\
\hline $\begin{array}{l}\text { Number of drugs acting on the } \\
\text { ACE2 receptor and/or DDP4 } \ddagger\end{array}$ & & & $<0.001$ & & & 0.706 \\
\hline 0 & $17(56.7 \%)$ & $232(88.2 \%)$ & & $17(56.7 \%)$ & $17(56.7 \%)$ & \\
\hline 1 & $9(30.0 \%)$ & $27(10.3 \%)$ & & $9(30.0 \%)$ & $11(36.7 \%)$ & \\
\hline 2 & $3(10.0 \%)$ & $3(1.1 \%)$ & & $3(10.0 \%)$ & $2(6.7 \%)$ & \\
\hline 3 & $1(3.3 \%)$ & $1(0.4 \%)$ & & $1(3.3 \%)$ & $0(0.0 \%)$ & \\
\hline
\end{tabular}

* ECOG, Eastern Cooperative Oncology Group performance status; SpO2, Pulse Oximeter Oxygen Saturation; ACE, angiotensin converting enzyme; DDP4, dipeptidyl peptidase $4 .{ }^{\dagger}$ Includes ibuprofen, angiotensin II receptor blockers, and thiazolidinediones. ${ }^{\ddagger}$ Includes ibuprofen, angiotensin II receptor blockers, thiazolidinediones, and dipeptidyl peptidase-4 inhibitor. 
Table A5. Cox regression of risk factors for progression-free survival of COVID-19 patients *

\begin{tabular}{|c|c|c|c|}
\hline & HR & $95 \% \mathrm{CI}$ & $p$ \\
\hline Age & 1.061 & $1.04-1.09$ & $<0.001$ \\
\hline Male ex & 0.7284 & $0.36-1.46$ & 0.370 \\
\hline Healthcare-acquired infection & 6.214 & $1.90-20.30$ & 0.002 \\
\hline ECOG performance status & 3.373 & $1.89-6.02$ & $<0.001$ \\
\hline Time from disease confirmation to admission, days & 0.9714 & $0.87-1.09$ & 0.607 \\
\hline Time from symptom onset to admission, days & 1.02 & $0.98-1.06$ & 0.330 \\
\hline Time from symptom onset to confirmation, days & 1.025 & $0.97-1.08$ & 0.344 \\
\hline \multicolumn{4}{|l|}{ Initial symptoms } \\
\hline Asymptomatic & 0.169 & $0.05-0.55$ & 0.003 \\
\hline Productive cough & 1.85 & $0.95-3.59$ & 0.069 \\
\hline Fever & 4.139 & $2.14-7.99$ & $<0.001$ \\
\hline Cough & 0.3916 & $0.14-1.11$ & 0.077 \\
\hline Headache & 3.44 & $1.78-6.64$ & $<0.001$ \\
\hline Myalgia or fatigue & 2.704 & $1.38-5.29$ & 0.004 \\
\hline Chill & 5.983 & $3.11-11.52$ & $<0.001$ \\
\hline Sore throat & 1.467 & $0.64-3.35$ & 0.363 \\
\hline Rhinorrhea & 0.8683 & $0.31-2.46$ & 0.790 \\
\hline Dyspnea & 6.065 & $2.92-12.59$ & $<0.001$ \\
\hline Diarrhea & 4.33 & $1.90-9.90$ & 0.001 \\
\hline Nausea or vomiting & 3.423 & $1.05-11.17$ & 0.041 \\
\hline Chest pain & 5.574 & $1.97-15.79$ & 0.001 \\
\hline \multicolumn{4}{|l|}{ Initial signs } \\
\hline Body temperature, ${ }^{\circ} \mathrm{C}$ & 4.033 & $2.74-5.94$ & $<0.001$ \\
\hline Systolic blood pressure, $\mathrm{mm} \mathrm{Hg}$ & 1.01 & $0.99-1.03$ & 0.402 \\
\hline Diastolic blood pressure, $\mathrm{mm} \mathrm{Hg}$ & 1.027 & $1.00-1.06$ & 0.046 \\
\hline Pulse rate, beats/min & 1.035 & $1.01-1.06$ & 0.007 \\
\hline Respiratory rate, beats/min & 1.106 & $0.94-1.30$ & 0.217 \\
\hline $\mathrm{SpO} 2, \%$ & 0.7119 & $0.56-0.90$ & 0.005 \\
\hline \multicolumn{4}{|l|}{ Comorbidities } \\
\hline Hypertension & 3.56 & $1.67-7.58$ & 0.001 \\
\hline Diabetes mellitus & 6.59 & $3.17-13.69$ & $<0.001$ \\
\hline Allergic disease & 0.1782 & $0.02-1.30$ & 0.089 \\
\hline Chronic lung disease & 1.473 & $0.45-4.80$ & 0.521 \\
\hline Peripheral vascular disease & 0.6158 & $0.084-4.50$ & 0.633 \\
\hline Cerebrovascular disease & 4.71 & $1.13-19.62$ & 0.033 \\
\hline Rheumatic disease & 4.95 & $0.68-36.20$ & 0.115 \\
\hline Congestive heart failure & 3.239 & $0.78-13.49$ & 0.107 \\
\hline \multicolumn{4}{|l|}{ Prior history of drug use } \\
\hline Ibuprofen & 2.722 & $1.13-6.54$ & 0.025 \\
\hline Angiotensin II receptor blocker & 4.261 & $1.77-10.25$ & 0.001 \\
\hline Calcium channel blocker & 3.251 & $1.26-8.37$ & 0.015 \\
\hline Beta blocker & 2.056 & $0.49-8.56$ & 0.322 \\
\hline Dipeptidyl peptidase- 4 inhibitor & 6.878 & $3.13-15.12$ & $<0.001$ \\
\hline Metformin & 6.196 & $2.58-14.91$ & $<0.001$ \\
\hline Sulfonylurea & 5.775 & $1.39-24.08$ & 0.016 \\
\hline Thiazolidinedione & 42.2 & $5.41-329.10$ & $<0.001$ \\
\hline Isosorbide & 17.69 & $2.35-132.90$ & 0.005 \\
\hline Statin & 4.46 & $1.86-10.73$ & 0.001 \\
\hline KCDC classification I & 6.172 & $3.87-9.85$ & $<0.001$ \\
\hline KCDC classification II & 2.137 & $1.54-2.97$ & $<0.001$ \\
\hline CT score & 1.191 & $1.13-1.25$ & $<0.001$ \\
\hline MuLBSTA & 1.253 & $1.15-1.37$ & $<0.001$ \\
\hline CURB65 & 4.142 & $1.97-8.73$ & $<0.001$ \\
\hline Pneumonia severity index & 2.539 & $1.74-3.70$ & $<0.001$ \\
\hline Age-adjusted Charlson comorbidity index & 1.608 & $1.33-1.94$ & $<0.001$ \\
\hline
\end{tabular}

* HR, hazard ratio; CI, confidence interval; NA; ECOG, Eastern Cooperative Oncology Group performance status; SpO2, Pulse Oximeter Oxygen Saturation; KCDC, Korea Centers for Disease Control and Prevention; $\mathrm{CT}$, computed tomography; MuLBSTA, Multilobular infiltration, hypo-Lymphocytosis, Bacterial coinfection, Smoking history, hyper-Tension and Age; CURB65, Confusion, Urea, Respiratory rate, Blood pressure plus age $\geq 65$ year. 


\section{References}

1. Richman, D.D.; Whitley, R.J.; Hayden, F.G. Clinical Virology, 4th ed.; ASM Press: Washington, DC, USA, 2016.

2. Lu, H.; Stratton, C.W.; Tang, Y.W. Outbreak of pneumonia of unknown etiology in Wuhan, China: The mystery and the miracle. J. Med. Virol. 2020, 92, 401-402. [CrossRef] [PubMed]

3. Hui, D.S.; Azhar, I.E.; Madani, T.A.; Ntoumi, F.; Kock, R.; Dar, O.; Ippolito, G.; McHugh, T.D.; Memish, Z.A.; Drosten, C.; et al. The continuing 2019-nCoV epidemic threat of novel coronaviruses to global health-The latest 2019 novel coronavirus outbreak in Wuhan, China. Int. J. Infect. Dis. 2020, 91, 264-266. [CrossRef] [PubMed]

4. Huang, C.; Wang, Y.; Li, X.; Ren, L.; Zhao, J.; Hu, Y.; Zhang, L.; Fan, G.; Xu, J.; Gu, X.; et al. Clinical features of patients infected with 2019 novel coronavirus in Wuhan, China. Lancet 2020, 395, 497-506. [CrossRef]

5. Coronavirus Disease (COVID-2019) Situation Reports; World Health Organization (WHO): Geneva, Switzerland, 2020.

6. Wang, D.; Hu, B.; Hu, C.; Zhu, F.; Liu, X.; Zhang, J.; Wang, B.; Xiang,H.; Cheng, Z.; Xiong, Y.; et al. Clinical characteristics of 138 hospitalized patients with 2019 novel coronavirus-infected pneumonia in Wuhan, China. JAMA 2020. [CrossRef]

7. Chen, N.; Zhou, M.; Dong, X.; Qu, J.; Gong, F.; Han, Y.; Qiu, Y.; Wang, J.; Liu, Y.; Wei, Y.; et al. Epidemiological and clinical characteristics of 99 cases of 2019 novel coronavirus pneumonia in Wuhan, China: A descriptive study. Lancet 2020, 395, 507-513. [CrossRef]

8. Deng, S.Q.; Peng, H.J. Characteristics of and public health responses to the coronavirus disease 2019 outbreak in China. J. Clin. Med. 2020, 9, 575. [CrossRef] [PubMed]

9. Kim, J.Y.; Choe, P.G.; Oh, Y.; Oh, K.J.; Kim, J.; Park, S.J.; Park, J.H.; Na, H.K.; Oh, M.D. The first case of 2019 novel coronavirus pneumonia imported into Korea from Wuhan, China: Implication for infection prevention and control measures. J. Korean Med. Sci. 2020, 35, e61. [CrossRef]

10. The Update of COVID-19 in Korea as of 23 February; Korea Centers for Disease Control and Prevention (KCDC): Cheongju, Korea, 2020. Available online: https://www.cdc.go.kr/board/board.es?mid=a30402000000\&bid= 0030\&act=view\&list_no=366307\&tag $=\& n$ Page $=1$ (accessed on 23 February 2020).

11. Frequently Asked Questions for KCDC on COVID-19; Korea Centers for Disease Control and Prevention (KCDC): Cheongju, Korea, 2020; Available online: https://www.cdc.go.kr/board/board.es?mid=a30402000000\&bid= 0030 (accessed on 7 April 2020).

12. Zhang, J.J.; Dong, X.; Cao, Y.Y.; Yuan, Y.D.; Yang, Y.B.; Yan, Y.Q.; Akdis, C.A.; Gao, Y.D. Clinical characteristics of 140 patients infected with SARS-CoV-2 in Wuhan, China. Allergy 2020. [CrossRef]

13. Tian, S.; Hu, N.; Lou, J.; Chen, K.; Kang, X.; Xiang, Z.; Chen, H.; Wang, D.; Liu, N.; Liu, D.; et al. Characteristics of COVID-19 infection in Beijing. J. Infect. 2020, 80, 401-406. [CrossRef] [PubMed]

14. Young, B.E.; Ong, S.W.X.; Kalimuddin, S.; Low, J.G.; Tan, S.Y.; Loh, J.; Ng, O.T.; Marimuthu, K.; Ang, L.W.; Mak, T.M.; et al. Epidemiologic features and clinical course of patients infected with SARS-CoV-2 in Singapore. JAMA 2020. [CrossRef]

15. McMichael, T.M.; Currie, D.W.; Clark, S.; Pogosjans, S.; Kay, M.; Schwartz, N.G.; Lewis, J.; Baer, A.; Kawakami, V.; Lukoff, M.D.; et al. Epidemiology of COVID-19 in a long-term care facility in king county, Washington. N. Engl. J. Med. 2020. [CrossRef] [PubMed]

16. Charlson, M.E.; Pompei, P.; Ales, K.L.; MacKenzie, C.R. A new method of classifying prognostic comorbidity in longitudinal studies: Development and validation. J. Chronic Dis 1987, 40, 373-383. [CrossRef]

17. Zubrod, C.G.; Schneiderman, M.; Frei, E., III; Brindley, C.; Gold, G.L.; Shnider, B.; Oviedo, R.; Gorman, J.; Jones, R., Jr.; Jonsson, U. Appraisal of methods for the study of chemotherapy of cancer in man: Comparative therapeutic trial of nitrogen mustard and triethylene thiophosphoramide. J. Chronic Dis. 1960, 11, 7-33. [CrossRef]

18. Guo, L.; Wei, D.; Zhang, X.; Wu, Y.; Li, Q.; Zhou, M.; Qu, J. Clinical features predicting mortality risk in patients with viral pneumonia: The mulbsta score. Front. Microbiol. 2019, 10, 2752. [CrossRef]

19. Fine, M.J.; Auble, T.E.; Yealy, D.M.; Hanusa, B.H.; Weissfeld, L.A.; Singer, D.E.; Coley, C.M.; Marrie, T.J.; Kapoor, W.N. A prediction rule to identify low-risk patients with community-acquired pneumonia. N. Engl. J. Med. 1997, 336, 243-250. [CrossRef] 
20. Lim, W.S.; van der Eerden, M.M.; Laing, R.; Boersma, W.G.; Karalus, N.; Town, G.I.; Lewis, S.A.; Macfarlane, J.T. Defining community acquired pneumonia severity on presentation to hospital: An international derivation and validation study. Thorax 2003, 58, 377-382. [CrossRef]

21. Xiong, Y.; Sun, D.; Liu, Y.; Fan, Y.; Zhao, L.; Li, X.; Zhu, W. Clinical and high-resolution CT features of the COVID-19 infection: Comparison of the initial and follow-up changes. Investig. Radiol. 2020. [CrossRef] [PubMed]

22. Li, K.; Wu, J.; Wu, F.; Guo, D.; Chen, L.; Fang, Z.; Li, C. The clinical and chest CT features associated with severe and critical COVID-19 pneumonia. Investig. Radiol. 2020. [CrossRef]

23. Zhao, W.; Zhong, Z.; Xie, X.; Yu, Q.; Liu, J. Relation between chest CT findings and clinical conditions of coronavirus disease (COVID-19) pneumonia: A multicenter study. AJR Am. J. Roentgenol. 2020. [CrossRef]

24. Wyss, R.; Girman, C.J.; LoCasale, R.J.; Brookhart, A.M.; Sturmer, T. Variable selection for propensity score models when estimating treatment effects on multiple outcomes: A simulation study. Pharmacoepidemiol. Drug Saf. 2013, 22, 77-85. [CrossRef] [PubMed]

25. Hill, J. Discussion of research using propensity-score matching: Comments on "A critical appraisal of propensity-score matching in the medical literature between 1996 and 2003" by peter austin, statistics in medicine. Stat. Med. 2008, 27, 2055-2061. [CrossRef]

26. Shi, Y.; Yu, X.; Zhao, H.; Wang, H.; Zhao, R.; Sheng, J. Host susceptibility to severe COVID-19 and establishment of a host risk score: Findings of 487 cases outside Wuhan. Crit. Care 2020, 24, 108. [CrossRef]

27. Guan, W.J.; Ni, Z.Y.; Hu, Y.; Liang, W.H.; Ou, C.Q.; He, J.X.; Liu, L.; Shan, H.; Lei, C.L.; Hui, D.S.C.; et al. Clinical characteristics of coronavirus disease 2019 in China. N. Engl. J. Med. 2020. [CrossRef] [PubMed]

28. Peng, Y.D.; Meng, K.; Guan, H.Q.; Leng, L.; Zhu, R.R.; Wang, B.Y.; He, M.A.; Cheng, L.X.; Huang, K.; Zeng, Q.T. Clinical characteristics and outcomes of 112 cardiovascular disease patients infected by 2019-nCoV. Zhonghua Xin Xue Guan Bing Za Zhi 2020, 48, E004.

29. Xu, X.; Chen, P.; Wang, J.; Feng, J.; Zhou, H.; Li, X.; Zhong, W.; Hao, P. Evolution of the novel coronavirus from the ongoing Wuhan outbreak and modeling of its spike protein for risk of human transmission. Sci. China Life Sci. 2020, 63, 457-460. [CrossRef]

30. Wan, Y.; Shang, J.; Graham, R.; Baric, R.S.; Li, F. Receptor recognition by the novel coronavirus from Wuhan: An analysis based on decade-long structural studies of SARS coronavirus. J. Virol. 2020, 94. [CrossRef]

31. Li, L.Q.; Huang, T.; Wang, Y.Q.; Wang, Z.P.; Liang, Y.; Huang, T.B.; Zhang, H.Y.; Sun, W.; Wang, Y. COVID-19 patients' clinical characteristics, discharge rate, and fatality rate of meta-analysis. J. Med. Virol. 2020. [CrossRef] [PubMed]

32. Verdecchia, P.; Angeli, F.; Mazzotta, G.; Ambrosio, G.; Reboldi, G. Angiotensin converting enzyme inhibitors and angiotensin receptor blockers in the treatment of hypertension: Should they be used together? Curr. Vasc. Pharmacol. 2010, 8, 742-746. [CrossRef]

33. Ferrario, C.M.; Jessup, J.; Chappell, M.C.; Averill, D.B.; Brosnihan, K.B.; Tallant, E.A.; Diz, D.I.; Gallagher, P.E. Effect of angiotensin-converting enzyme inhibition and angiotensin ii receptor blockers on cardiac angiotensin-converting enzyme 2. Circulation 2005, 111, 2605-2610. [CrossRef] [PubMed]

34. Vuille-dit-Bille, R.N.; Camargo, S.M.; Emmenegger, L.; Sasse, T.; Kummer, E.; Jando, J.; Hamie, Q.M.; Meier, C.F.; Hunziker, S.; Forras-Kaufmann, Z.; et al. Human intestine luminal ace2 and amino acid transporter expression increased by ace-inhibitors. Amino Acids 2015, 47, 693-705. [CrossRef]

35. Gurwitz, D. Angiotensin receptor blockers as tentative SARS-CoV-2 therapeutics. Drug Dev. Res. 2020. [CrossRef] [PubMed]

36. Qiao, W.; Wang, C.; Chen, B.; Zhang, F.; Liu, Y.; Lu, Q.; Guo, H.; Yan, C.; Sun, H.; Hu, G.; et al. Ibuprofen attenuates cardiac fibrosis in streptozotocin-induced diabetic rats. Cardiology 2015, 131, 97-106. [CrossRef] [PubMed]

37. Vankadari, N.; Wilce, J.A. Emerging Wuhan (COVID-19) coronavirus: Glycan shield and structure prediction of spike glycoprotein and its interaction with human CD26. Emerg. Microbes Infect. 2020, 9, 601-604. [CrossRef] [PubMed]

38. Vaduganathan, M.; Vardeny, O.; Michel, T.; McMurray, J.J.V.; Pfeffer, M.A.; Solomon, S.D. Renin-angiotensinaldosterone system inhibitors in patients with COVID-19. N. Engl. J. Med. 2020. [CrossRef]

39. Cao, B.; Wang, Y.; Wen, D.; Liu, W.; Wang, J.; Fan, G.; Ruan, L.; Song, B.; Cai, Y.; Wei, M.; et al. A trial of lopinavir-ritonavir in adults hospitalized with severe COVID-19. N. Engl. J. Med. 2020. [CrossRef]

(C) 2020 by the authors. Licensee MDPI, Basel, Switzerland. This article is an open access article distributed under the terms and conditions of the Creative Commons Attribution (CC BY) license (http://creativecommons.org/licenses/by/4.0/). 